40922693-file00.docx

\title{
1 PDIP38 is a novel adaptor-like modulator of the mitochondrial AAA+ protease 2 CLPXP
}

3

4 Philip R. Strack ${ }^{1}$, Erica J. Brodie ${ }^{1, \S}$, Hanmiao Zhan ${ }^{1}$, Verena J. Schuenemann ${ }^{2, \dagger}$, Liz J. Valente ${ }^{1, f}$, 5 Tamanna Saiyed ${ }^{1}$, Kornelius Zeth ${ }^{2,3}$, Kaye N. Truscott ${ }^{1 *}$ and David A. Dougan ${ }^{1 *}$

6

7

$8{ }^{1}$ Department of Biochemistry and Genetics, La Trobe Institute for Molecular Science, La Trobe 9 University, Melbourne, Victoria, 3086, Australia.

$10{ }^{2}$ Department of Protein Evolution, Max-Planck-Institute for Developmental Biology, Tübingen, 11 Germany.

$12{ }^{3}$ Department of Science and Environment, Roskilde University, DK-4000, Denmark.

13 "These authors contributed equally to this work

14

$15{ }^{f}$ Present address: Department of Radiation Oncology, Stanford University School of Medicine, 16 Stanford, CA 94305, USA.

17 § Present address: Department of Immunology and Pathology, Monash University, Melbourne, 3004, 18 Victoria, Australia

$19{ }^{\dagger}$ Present address: Institute of Evolutionary Medicine, University of Zurich, Zurich, Switzerland. 20 21

22 Correspondence and requests for materials should be addressed to K.N.T. (email: 23 k.truscott@latrobe.edu.au) or D.A.D. (email: d.dougan@latrobe.edu.au). 
40922693-file00.docx

\section{Summary}

27 Polymerase $\delta$ interacting protein of $38 \mathrm{kDa}$ (PDIP38) was originally identified in a yeast two hybrid 28 screen as an interacting protein of DNA polymerase delta, more than a decade ago. Since this time several subcellular locations have been reported and hence its function remains controversial. Our current understanding of PDIP38 function has also been hampered by a lack of detailed biochemical or structural analysis of this protein. Here we show, that human PDIP38 is directed to the mitochondrion, where it resides in the matrix compartment, together with its partner protein CLPX. PDIP38 is a bifunctional protein, composed of two conserved domains separated by an $\alpha$-helical hinge region (or middle domain). The N-terminal (YccV-like) domain of PDIP38 forms an SH3-like $\beta$-barrel, which interacts specifically with CLPX, via the adaptor docking loop within the Nterminal Zinc binding domain (ZBD) of CLPX. In contrast, the C-terminal (DUF525) domain forms an Immunoglobin-like $\beta$-sandwich fold, which contains a highly conserved hydrophobic groove. Based on the physicochemical properties of this groove, we propose that PDIP38 is required for the recognition (and delivery to CLPXP) of proteins bearing specific hydrophobic degrons, potentially located at the termini of the target protein. Significantly, interaction with PDIP38 stabilizes the steady state levels of CLPX in vivo. Consistent with these data, PDIP38 inhibits the LONMmediated turnover of CLPX in vitro. Collectively, our findings shed new light on the mechanistic and functional significance of PDIP38, indicating that in contrast to its initial identification as a nuclear protein, PIDP38 is a bona fide mitochondrial adaptor protein for the CLPXP protease.

Keywords: PDIP38, CLPX, adaptor, structure, mitochondrial proteostasis. 


\section{3-file00.docx}

\section{Introduction}

Polymerase $\delta$ interacting protein of $38 \mathrm{kDa}$ (PDIP38, also known as POLDIP2 and mitogenin) was originally discovered through a yeast two hybrid screen, as a p50 (subunit of DNA polymerase delta) interacting protein ${ }^{1}$. Subsequently, PDIP38 has been identified in the nucleus, where it is proposed to play a role in DNA repair ${ }^{2-5}$. It has also been located in the cytoplasm and the plasma membrane ${ }^{2,4-6}$ where it has been implicated in a variety of cellular functions, ranging from cell proliferation ${ }^{7}$, to regulation of the extracellular matrix ${ }^{8}$, oxidative signalling and cell migration ${ }^{9}$, Tau aggregation ${ }^{10}$ and cancer ${ }^{11}$. Despite the extensive repertoire of putative physiological functions for PDIP38, few of the proposed interactions have been biochemically validated. Indeed, in contrast to its proposed role in the nucleus, there is growing evidence to suggest that PDIP38, through its interaction with CLPX, forms part of the mitochondrial protein homeostasis (proteostasis) network 12-14. However, currently little is known about the structure or function of PDIP38, its mechanism of action or its role in mitochondrial proteostasis.

Proteostasis involves the constant surveillance and maintenance of the proteome, from a proteins synthesis on a ribosome, through its folding and transport to the correct subcellular location and ultimately its removal from the cell in a timely manner ${ }^{15}$. This process is maintained by a network of proteins, which includes proteolytic machines and their associated cofactors that are responsible for the timely recognition and removal of damaged or unwanted proteins. In eukaryotes, the major non-lysosomal degradation pathway is mediated by the Ubiquitin (Ub) Proteasome System (UPS), which is responsible for the recognition (by a Ub ligase) of specific degradation signals (degrons) within a target protein, resulting in the conjugation of Ub (usually in the form of polyUb) to a Lys residue within the target. Ultimately, the tagged protein is processed into short peptides by a single ATP-dependent protease (the $26 \mathrm{~S}$ proteasome). The broad specificity of this system is achieved through the large number of $\mathrm{Ub}$ ligases ( 1,000 in mammals), which mediate recognition of different substrates. In contrast to the mammalian cytosol, protein degradation in eukaryotic organelles (similar to that in the bacterial cytosol) is mediated by several different ATP-dependent proteases, which function together with a handful of specialised adaptor proteins to enhance their substrate specificity ${ }^{16-18}$. Collectively these machines, regardless of their subcellular localisation, are referred to as AAA+ (ATPases associated with a variety of cellular activities) proteases ${ }^{19-22}$ as they are generally composed of two components, an unfoldase component belonging to the AAA+ superfamily and a peptidase component. In human mitochondria, five different AAA+ proteases have been discovered, two soluble matrix proteases; CLPXP (composed of two separate components; an unfoldase component CLPX and the peptidase component CLPP) and LONM (also known as LONP1) and three membrane bound proteases; an intermembrane space-AAA (i-AAA) 


\section{3-file00.docx}

83 protease and two forms of matrix-AAA (m-AAA) protease ${ }^{23}$. Although LONM is considered to be

84 the principal matrix quality control protease ${ }^{24-27}$ there is growing evidence that CLPXP also plays a 85 crucial role in mitochondrial proteostasis, contributing to heme regulation ${ }^{28-30}$, mitoribosomal 86 assembly ${ }^{31}$ and the selective turnover of ROS-damaged subunits of Complex I ${ }^{32}$. Consistently, 87 mutations in CLPP that cause Perrault syndrome 3 are linked to mitochondrial dysfunction ${ }^{33-35}$. 88 Moreover, targeted dysregulation of mitochondrial CLPP was also recently demonstrated to be 89 lethal to specific cancer cells ${ }^{36,37}$. Despite the emerging importance of this protease complex in 90 human mitochondria, our understanding of this machine and its mechanism of substrate recognition 91 is largely based on homologous prokaryotic systems.

Here we show, that human PDIP38 is imported into isolated mitochondria, where it co-localizes with its partner proteins CLPX (and CLPP) in the matrix compartment. Importantly, PDIP38 does not trigger dissociation of the CLPXP complex, but rather we propose that PDIP38 is a mitochondrial adaptor protein for the AAA+ protease, CLPXP. Consistent with its role as a CLPXadaptor protein, the structure of PDIP38 is composed of two domains, an N-terminal "YccV-like" domain which docks to an adaptor binding loop within the ZBD of CLPX, and a C-terminal DUF525-domain which forms an immunoglobulin-like fold bearing a putative substrate-binding groove. Significantly, the putative substrate-binding groove is lined with conserved hydrophobic residues and capped (at both ends) with conserved charged residues (basic at one end and acidic at

102 the other). We speculate that, this groove is responsible for the specific recognition of short 103 hydrophobic degrons, potentially located at the termini of a protein. Intriguingly, both domains have been found to recur throughout evolution (in bacteria, plants and humans), in select components of diverse protein degradation pathways. The $\mathrm{YccV}$ domain has been identified in bacterial (HspQ) and plant $(\mathrm{ClpF})$ proteins, which appears to act as an anti-adaptor of the N-recognin, ClpS and hence regulates the recognition and turnover of N-degron bearing substrates ${ }^{38,39}$. In contrast, the

108 ApaG domain has been identified in Fbox-only proteins such as Fbx3, where it is proposed to act as 109 a substrate recognition component. Consistent with this premise, we show that PDIP38 inhibits the 110 LONM-mediated turnover of CLPX in vitro and stabilizes the steady state levels of CLPX in vivo. 111 As such, PDIP38 regulates CLPXP activity, both directly (through the potential delivery of specific 112 substrates to CLPX for degradation by CLPP) and indirectly (through inhibition of the LONM113 mediated turnover of CLPX in vivo). 


\section{3-file00.docx}

\section{RESULTS}

\section{Human PDIP38 is a matrix localised mitochondrial protein}

117 To date, mammalian PDIP38 has been identified in several subcellular compartments, from the 118 plasma membrane to the nucleus and the mitochondrion ${ }^{1,6,12}$, and as such its subcellular localisation 119 is currently controversial. Therefore, to validate the sub-cellular localisation of human PDIP38, we 120 performed in vitro import assays into isolated mitochondria and mitochondrial fractionation 121 experiments (Fig. 1). Consistent with our identification of murine PDIP38 as a mitochondrial 122 protein ${ }^{12}$, radiolabelled human preprotein (pPDIP38) was imported into isolated mitochondria in a 123 membrane potential-dependent manner (Fig. 1a, compare lanes 5 and 6). Importantly the processed, 124 mature form of the protein (mPDIP38) was protected from cleavage by Proteinase K (Prot. K), 125 demonstrating that mPDIP38 was sequestered inside the mitochondrion. Next, we performed a sub126 mitochondrial fractionation of mitochondria isolated from HeLa cells coupled with a protease 127 protection assays using Prot. K. Consistent with the location of PDIP38 within the mitochondrial 128 matrix, and similar to a known matrix protein - CLPP, PDIP38 was protected from digestion by 129 Prot. K in both intact mitochondria (Fig. 1b, lanes 1-4) and mitoplast (Fig. 1b, lanes 5 - 8). As a 130 control, the outer membrane protein (TOM20) was digested by Prot. K under all conditions, while 131 the inner membrane protein (TIM23) was completely protected in intact mitochondria but sensitive 132 in mitoplast (Fig. 1b, lanes 5 - 8). Next, having established that human PDIP38 was indeed located 133 within the mitochondrial matrix we analysed the interaction between CLPX and PDIP38 in human 134 mitochondria by co-immunoprecipitation (co-IP). Initially, we examined the interaction of 135 endogenous CLPX (with endogenous PDIP38), using a PDIP38 specific antisera immobilised to 136 Protein A Sepharose (PAS). Consistent with a specific interaction between PDIP38 and CLPX,

137 CLPX was only recovered in the presence of anti-PDIP38 (Fig. 1c, lane 3 lower panel) and not in 138 the presence of the pre-immune sera (Fig. 1c, lane 2 lower panel). Next, to confirm this interaction 139 we performed the reverse co-IP, in which the anti-CLPX antisera was immobilised to PAS. 140 Consistent with the specific IP of CLPX with anti-PDIP38, the IP of CLPX using anti-CLPX 141 antisera also resulted in the specific co-IP of PDIP38 (Fig. 1d, lane 3 lower panel).

\section{The ZBD of CLPX is sufficient for interaction with PDIP38}

144 Next, to ensure that the interaction observed in vivo (between PDIP38 and CLPX) was direct, we 145 purified both components and examined the interaction in vitro. To do so, we generated a GST146 PDIP38 fusion protein in which PDIP38 was fused to the C-terminus of GST. Following expression 147 of GST-PDIP38, a soluble lysate (bearing overexpressed GST-PDIP38) was applied to Ni-NTA 148 agarose beads (either lacking or containing immobilised His ${ }_{10}$-tagged CLPX $\left(\mathrm{H}_{10} \mathrm{CLPX}\right.$, see Fig. 
40922693-file00.docx

149 2a). Following incubation of GST-PDIP38 with the beads, the specifically bound proteins were

150 eluted from the column with imidazole (Fig. 2b, lanes 4 and 7). Consistent with our identification

151 of PDIP38 as a novel CLPX interacting protein (Fig. 1), GST-PDIP38 was only recovered in the

152 presence of immobilised $\mathrm{H}_{10}$ CLPX (Fig. 2b, lanes 7) and not in the absence of an immobilised

153 protein (Fig. 2b, lanes 4).

154 Next, we asked the question how does the CLPX-PDIP38 complex form? Initially, we speculated

155 that PDIP38 might be an adaptor protein of human CLPX, and as such would likely bind to an

156 accessory domain of CLPX, as is the case for several bacterial AAA+ adaptor proteins ${ }^{16,40-42}$.

157 Interestingly in contrast to bacterial ClpX homologs, human CLPX contains two accessory domains,

158 an N-terminal C4 type Zinc finger domain (often referred to as a Zinc binding domain (ZBD)) and

159 an additional domain that is inserted into the AAA module of CLPX, which is unique to eukaryotic

160 CLPX homologs (termed the E-domain ${ }^{43}$ ). Therefore, to identify which CLPX domain (or domains)

161 might be responsible for the interaction with PDIP38, we generated and purified $\mathrm{H}_{10}$-tagged

162 versions of both the ZBD (Fig. 2a, ZBD) and the E-domain (Fig. 2a, E) of CLPX. These proteins

163 were then immobilised to Ni-NTA agarose (as described above) and a soluble lysate bearing GST-

164 PDIP38 was applied to the appropriate columns (Fig. 2c, lane 2, 5 and 8). Consistent with the data

165 above, in which PDIP38 was recovered in the presence of full-length CLPX, PDIP38 was

166 specifically co-eluted from the column containing immobilised ZBD (Fig. 2c, lane 10) and not from

167 the column containing immobilised E-domain (Fig. 2c, lane 7). Collectively these data demonstrate

168 that PDIP38 interacts specifically with the ZBD of CLPX. Next, we examined the stoichiometry of

169 the interaction between PDIP38 and the N-domain. To address this question, we purified full-length

170 PDIP38 (containing an N-terminal $\mathrm{H}_{10}$-tag) and monitored complex formation by size exclusion

171 chromatography, using Superose 12. Both proteins alone formed a monodispersed peak on gel

172 filtration, PDIP38 (alone) eluted in a single peak (Fig. 2d, upper panel), with an apparent molecular

173 weight of $\sim 39 \mathrm{kDa}$ (consistent with a monomeric protein in solution), while in contrast the ZBD of

174 CLPX (Fig. 2d, middle panel) eluted at $\sim 15 \mathrm{ml}$, (which is consistent with an apparent molecular

175 weight of $\sim 24 \mathrm{kDa}$ and hence likely a homodimer of $\left.\mathrm{CLPX}_{\mathrm{ZBD}}\right)$. Consistent with the pull-down

176 (Fig. 2d), PDIP38 formed a stable complex with CLPX $_{\mathrm{ZBD}}$, which based on the elution volume of

177 the complex $(\sim 13.6 \mathrm{ml}$, equivalent to $\sim 49 \mathrm{kDa})$ likely forms a heterodimeric complex composed of

178 one subunit of each protein (Fig. 2d, lower panel).

179

180 PDIP38 is composed of two domains - only the NTD is required for interaction with CLPX

181 Having determined which domain of CLPX is required for interaction with PDIP38, we next

182 examined the domain structure of PDIP38 with the aim of defining which region or regions in

183 PDIP38 are required for interaction with the ZBD of CLPX. Initially we used a bioinformatic 


\section{3-file00.docx}

184 approach to determine the domain structure of PDIP38 ${ }^{44}$. This analysis revealed that PDIP38 is composed of two domains, a large YccV-like N-terminal domain (residues 52 - 234) and a smaller

186 C-terminal domain (residues 235 - 368) of unknown function (DUF525). Intriguingly, both

187 domains have been identified in proteins that have been implicated in a variety of protein degradation pathways from bacteria to plants and humans. The YccV-like domain is present in the F-box protein $\mathrm{Fbx} 21 / \mathrm{FbxO} 21$, which was recently demonstrated to form an integral component of the SCF (Skip-Cullin-Fbox) Ubiquitin (Ub) ligase complex required for the turnover of EID1 ${ }^{45,46}$. This domain was also recently identified in a unique component (termed $\mathrm{ClpF}$ ), which is proposed

192 to form part of the Clp protease machinery in the chloroplast of Arabidopsis thaliana ${ }^{38}$. In this case,

193 ClpF was shown to form a binary complex with ClpS1, a putative adaptor protein (or N-recognin) 194 that is required for the recognition of substrate proteins bearing specific N-degrons ${ }^{47,48}$. 195 Remarkably, bacterial YccV (recently renamed HspQ) was also shown to regulate protein turnover 196 via two proteolytic system, activating Lon-mediated turnover and inhibiting ClpS-mediated degradation of N-degron substrates ${ }^{39,49}$. Based on the bioinformatic analysis of PDIP38, we initially generated GST-fusion proteins of each domain, however unexpectedly neither fusion protein was soluble. Therefore, in order to identify a functional boundary of the proposed domains we performed limited proteolysis of mature PDIP38, using thermolysin (Fig. 3a). This approach revealed that PDIP38 was indeed composed of two stable domains (Fig. 3a, f1 and f2). However, based on the transient appearance of two intermediate fragments (f1' and f2'), these domains are likely separated by an exposed, flexible linker. To identify the boundary of these two domains we performed six rounds of Edman degradation on fragment $\mathrm{f1}$ (FLANHD). This defined $\mathrm{f} 1$ as the Cterminal DUF525 domain and identified the boundary of this domain as F157. Armed with this information we generated two additional GST-fusion proteins, GST-PDIP38 ${ }_{\mathrm{N}}(\mathbf{F i g}$. 3b) in which the N-terminal domain of PDIP38 (residues 52 to 153) was fused to the C-terminus of GST and GSTPDIP38 (see Fig. 3b) in which the C-terminal domain of PDIP38 (residues 157 to 368) was fused to C-terminus of GST (GST-PDIP38 $)$. To determine which domain was required for docking to CLPX we performed a series of pull-down assays, in which $\mathrm{H}_{10}$ CLPX was immobilised to NiNTAagarose beads and then incubated with a bacterial cell lysate containing either overexpressed GST-

212 PDIP38, GST-PDIP38 ${ }_{\mathrm{N}}$ or GST-PDIP38 (Fig. 3c, lanes 2, 4 and 6 respectively). As a control, the 213 different GST-PDIP38 fusion proteins were also incubated with Ni-NTA-agarose beads lacking 214 immobilised protein (Fig. 3c, lanes 3, 5 and 7, respectively). As expected, and consistent with 215 Figure $2 \mathrm{~b}$, full length GST-PDIP38 was specifically eluted from the column containing immobilised $216 \mathrm{H}_{10}$ CLPX (Fig. 3c, lanes 2). Significantly, deletion of the N-domain of PDIP38 (GST-PDIP38C) 217 was sufficient to prevent any specific interaction between the two proteins (Fig. 3c, compare lanes 6 218 and 7). Consistent with these results, the N-domain of PDIP38 alone was sufficient for the 
40922693-file00.docx

interaction with CLPX as the GST-PDIP38 $8_{\mathrm{N}}$ fusion was specifically recovered from NiNTA-agarose beads containing immobilised CLPX (Fig. 3c, lanes 4) and not from beads lacking immobilised protein (Fig. 3c, lanes 5). Taken together these results demonstrate that the N-terminal YccV-like domain of PDIP38 specifically docks to the ZBD of CLPX.

\section{PDIP38 docks to the "adaptor binding" loop within the NTD of CLPX to regulate turnover of a model substrate}

Next, we examined the consequence of PDIP38 docking to CLPX, i.e. is PDIP38 a substrate or an adaptor protein of CLPX, or does PDIP38 trigger dissociation of the CLPXP complex? To determine if PDIP38 is a substrate of CLPXP, we monitored the stability of PDIP38 in vitro, in the presence of active CLPXP (Fig. 4a). Given that substrate recognition by many Clp-proteases is generally mediated by degrons located at either the $\mathrm{N}$ - or C-termini ${ }^{50}$, we generated an untagged version of PDIP38, using the Ub-fusion system ${ }^{51}$. As a control, to ensure that human CLPXP was active, we monitored the turnover of the model unfolded protein (casein), a well-characterized CLPX substrate ${ }^{12,52}$. Significantly, in contrast to the rapid CLPXP-mediated turnover of FITCcasein (Fig. 4a, middle panel), the levels of untagged PDIP38 remained unchanged throughout the time course of the experiment (Fig. 4a, upper panel). These data clearly demonstrate that mature untagged PDIP38 is not a substrate of the CLPXP protease, but rather is either an adaptor protein for human $\operatorname{CLPX}(\mathrm{P})$ or alternatively a protein "switch" that triggers dissociation of CLPP from the CLPXP complex. In order to address the second possibility and determine if PDIP38 is able to modulate the specificity of CLPXP, we next monitored the CLPXP-mediated turnover of FITClabelled casein in the absence and presence of PDIP38 (Fig. 4a). Significantly, the addition of PDIP38 exhibited contrasting effects on the turnover of different forms of FITC-casein, specifically the CLPXP-mediated turnover of $\alpha_{\mathrm{S}_{2}}$ casein was inhibited by PDIP38 (Fig. 4a, lower panel), in a concentration-dependent manner (Supplementary Fig. 1), while the turnover of $\kappa$-casein was unaffected by the presence of PDIP38 (Fig. 4a, lower panel, Supplementary Fig. 1, black bars). Collectively these data suggest that PDIP38 was able to specifically inhibit the turnover of one substrate without affecting the turnover of another, demonstrating that PDIP38 does not trigger dissociation of CLPX from CLPP. In addition, PDIP38 itself was not a substrate of the CLPXP machine, suggesting that PDIP38 exhibits adaptor-like activity. To further investigate the possibility that PDIP38 is a CLPX adaptor protein we compared the ZBD of human CLPX with several other ClpX homologs, from both bacterial and eukaryotic species. In particular we focused on the known adaptor-docking region (Fig. 4c, adaptor binding loop). Despite considerable sequence conservation across the ZBD of bacterial and eukaryotic homologs, one region of the ZBD - the "adaptor binding loop" - diverged. This part of the domain was highly conserved amongst either eukaryotic 


\section{3-file00.docx}

254

255

256

257

258

259

260

261

262

263

264

265

266

267

268

269

270

271

272

273

274

275

276

277

278

279

280

281

282

283

284

285

286

287

288

(or bacterial) species but poorly conserved across the two kingdoms (Fig. 4c and 4d). Therefore, we hypothesized that this region may have coevolved with a new adaptor protein (i.e. PDIP38). To test the idea that the "adaptor binding loop" within the ZBD of human CLPX is required for docking to the putative adaptor protein PDIP38, we examined the ability of Escherichia coli ClpX (ecClpX) ZBD (ecZBD) to interact with human PDIP38 (Fig. 4b). Remarkably, in contrast to human ZBD (Fig. 4b, lane 1) which bound to PDIP38, ecZBD failed to interact with PDIP38 at all (Fig. 4b, lane 3). Next to confirm that the proposed adaptor binding loop was indeed the site of PDIP38 interaction in human CLPX we replaced the putative adaptor-docking region (residues 120 to 123; SSTR) with AAAA in both full-length CLPX (here referred to as CLPX $4 \mathrm{~A}$ ) and in the ZBD of CLPX (here referred to as $\mathrm{ZBD}_{4 \mathrm{~A}}$ ). Consistent with the loss of binding of PDIP38 to ecZBD, the recovery of untagged PDIP38 to either immobilised $\mathrm{ZBD}_{4 \mathrm{~A}}$ (Fig. 4e, lane 3) or CLPX $4 \mathrm{~A}$ (Fig. 4e, lane 7) was completely abolished, when compared to wild type ZBD (Fig. 4e, lane 1) or CLPX (Fig. 4e, lane 5). Collectively these data suggest that the "adaptor binding loop" within the ZBD of CLPX performs a conserved function in both bacterial and eukaryotic homologs of CLPX. Specifically, the ZBD of human CLPX forms a crucial docking platform for interaction with the putative adaptor protein PDIP38. This interaction prevents the CLPXP-mediated turnover of the model substrate, $\alpha_{\mathrm{S} 2}$-casein, while permitting the turnover of $\kappa$-casein $(\mathbf{F i g}$. 4a) suggesting that PDIP38 is a bona fide adaptor protein of mitochondrial CLPX.

Next, in order to determine the physiological function of PDIP38, we took two complementary approaches. In the first approach, we attempted to isolate PDIP38 interacting proteins. To do so, we knocked down PDIP38 expression in mammalian (HeLa) cells using siRNA with the aim of stabilising PDIP38-mediated substrates in vivo, before isolating the stabilised interacting proteins via pull-down. Although the knock down of PDIP38, using the PDIP38-specific siRNA (\#22994, Thermo Fisher) was successful (Fig. 5a, middle panel compare lanes $1-3$ with lanes $4-6$ ), this approach was largely unproductive in identifying specific PDIP38 interacting proteins. Nevertheless, when analysing the steady state levels of selected mitochondrial proteins in the knock down cells, we noticed that the levels of CLPX were reduced in HeLa cells transfected with the PDIP38specific siRNA (Fig. 5a, top panel, lanes 1 - 3) when compared to the levels of CLPX in HeLa cells transfected with a control siRNA (Fig. 5a, lanes 4 -6). Importantly, this change was specific for CLPX as the levels of CLPP (Fig. 5a, lower panel) and the cross-reactive band (Fig. 5a, middle panel, *) were unchanged by knock down of PDIP38. To validate these data, we examined the steady state level of CLPX using two additional PDIP38-specific siRNA's (s25055 and s25056) in comparison to an appropriate control siRNA (Supplementary Fig. 2). Significantly, the loss of CLPX (as a result of PDIP38 knock down) was specific, as the steady state levels of two unrelated 


\section{3-file00.docx}

289

290

291

292

293

294

295

296

297

298

299

300

301

302

303

304

305

306

307

308

309

310

311

312

313

314

315

316

317

318

319

320

321

322

323

proteins (i.e. mitochondrial SDHA or the cytosolic protein, GAPDH) were not affected (Supplementary Fig. 2b, lower panels).

From these data we speculated that PDIP38, similar to the ecClpA adaptor protein ecClpS (which protects its cognate unfoldase from auto-catalytic degradation in vivo ${ }^{40}$ ), inhibits the autocatalytic turnover of CLPX. To test this, we monitored the stability of CLPX in vitro, in the presence of CLPP, with or without the addition of PDIP38. Contrary to the idea that PDIP38 inhibited autocatalytic turnover of CLPX, the levels of CLPX (in vitro) remained unchanged in the presence of CLPP. Therefore, we hypothesized that the in vivo turnover of CLPX was mediated by an alternate mitochondrial matrix protease (i.e. LONM) and this turnover could be inhibited by PDIP38. To examine this possibility, we monitored the LONM-mediated degradation of CLPX in vitro, in the absence and presence of PDIP38 (Fig. 5b). Consistent with the idea that the levels of CLPX in vivo are controlled by the presence of PDIP38, CLPX was degraded by LONM in vitro (Fig. 5b, lanes 2 -7) with a half-life of $\sim 60$ min (Fig. 5c, open circles). Importantly, the LONM-mediated turnover of CLPX was inhibited by the addition of PDIP38 (Fig. 5b, lanes 8 - 13; Fig. 5c, filled circles). Significantly, the PDIP38-mediated inhibition of LONM was specific to the turnover of CLPX, as the LONM-mediated degradation of casein was unaffected by the addition of PDIP38 (Supplementary Fig. 3). Therefore, the inhibition of CLPX turnover is likely due to PDIP38 shielding the CLPX degron from interaction with LONM, which suggests that the CLPX degron is located within the ZBD of CLPX. Collectively, these data suggest that in the absence of PDIP38, the in vivo levels of CLPX may be regulated by LONM-mediated degradation.

Next, in order to better understand how substrate recognition by PDIP38 might occur, we crystalized mature PDIP38 (residues 52 to 368) and solved its structure by X-ray crystallography to $3.1 \AA$ resolution (refer to Supplementary Table 1 for statistics). Consistent with our biochemical analysis (see Fig. 3), the structure of PDIP38 is composed of two domains, an N-terminal YccV-like domain (residues 64 to 186) and a C-terminal DUF525 domain (residues 231 to 368), separated by a short middle domain or linker region (Fig. 6a). The N-terminal YccV-like domain forms an antiparallel $\beta$-sheet structure composed of six $\beta$-strands $(\beta 0-\beta 5-\beta 1-\beta 2-\beta 3-\beta 4)$, in which strands $\beta 0$ to $\beta 4$ are connected by loops and $\beta 4$ and $\beta 5$ is connected by a short $3^{10}$ helix (Fig. $6 \mathbf{b}$ and

Supplementary Fig. 4). In contrast to bacterial YccV (HspQ) homologs, PDIP38 contains a large insertion between $\beta 2$ and $\beta 3$, which forms an extended $\beta$-sheet that interacts with the proximal sheet of the DUF525 domain. Interestingly, this insertion is also present in other YccV-like proteins (including Human Fbx21), which lack the DUF525 domain. However, similar to PDIP38, Fbx21 contains an additional domain that is proposed to be involved in substrate-binding. Hence, we 


\section{3-file00.docx}

324 propose that similar to PDIP38, the extended $\beta 2 / \beta 3$ sheet in Fbx21 is likely involved in a stabilising 325 interaction with an associated substrate binding domain. In addition to the extension of the $\beta 2 / \beta 3$ 326 strands, PDIP38 also contains a unique insertion located between $\beta 3$ and $\beta 4$ (residues 143-166).

327 This insertion is not only exposed (as it was susceptible to partial proteolysis) but is also highly 328 flexible as it was not visible in the structure, presumably due to disorder. Based on the expected 329 location of this loop, suspended over the DUF525 domain, we speculate that the L4 loop regulates 330 substrate binding to the C-terminal domain. The linker region (or middle domain), which connects 331 the $\mathrm{N}$ - and C-terminal domains, is formed by a small $\mathrm{N}$-terminal $\alpha$-helix $(\alpha 1)$, a two-stranded anti332 parallel $\beta$-sheet ( $\beta 6$ and $\beta 7$ ) and a C-terminal $\alpha$-helix ( $\alpha 2)$. This domain makes extensive contact to 333 the N-terminal domain, wrapping around the domain, and likely forms a flexible hinge point for 334 movement of the C-terminal domain and hence delivery of bound cargo to the associated ATPase 335 component, CLPX. The C-terminal DUF525 domain (residues 231 to 368) forms an 336 Immunoglobin-like $\beta$-sandwich fold composed of two four-stranded antiparallel $\beta$-sheets. The 337 proximal sheet is composed of $\beta 8-\beta 9-\beta 10-\beta 13$, while the distal sheet is composed of strands $\beta 12-$ $338 \quad \beta 11-\beta 14-\beta 15$. Interestingly, although this domain exhibits only limited amino acid identity $(\sim 30 \%)$ 339 with bacterial ApaG proteins and eukaryotic F-box only proteins such as Fbx3, this group of 340 proteins share considerable structural homology (root-mean-squared deviation (RMSD) of $\sim 1.5$ A. 341 Consistent with our identification of PDIP38 as a putative substrate delivery factor for 342 mitochondrial CLPX, Fbx3 forms part of a SCF Ubiquitin ligase complex in which the DUF525 343 domain is proposed to be involved in substrate recognition ${ }^{53,54}$. Therefore, to gain further insight 344 into the function PDIP38 we examined the molecular surface of our structure (Fig. 6c). From this 345 analysis we identified a conserved hydrophobic groove, located between the two $\beta$-sheets of the C346 terminal domain, which is flanked by conserved charged residues at opposite ends of the groove 347 (Fig. 6d). To determine the significance of this groove we also examined the surface of 348 Xanthomonas axonopodis ApaG (PDB: 2F1E) and human Fbx3 (PDB:5HDW). Significantly, 349 despite the weak overall sequence similarity across this group of proteins, the physicochemical 350 properties of this groove are remarkably conserved across all three proteins (Fig. 6e and 6f, 351 Supplementary Fig. 5). Indeed, all but one of the 9 hydrophobic residues that line the hydrophobic 352 pocket and both of the charged residues that flank the groove are absolutely conserved from 353 bacteria to humans (see Supplementary Table 2 and Supplementary Fig. 5b). Furthermore, of the 354 absolutely conserved residues that found within this domain, approximately half of them are 355 clustered to the hydrophobic groove. Consistent with the notion that this conserved groove plays an 356 important role in substrate recognition, Chen and colleagues discovered a small molecule inhibitor 


\section{3-file00.docx}

of $\mathrm{Fbx} 3$, that docks into the conserved hydrophobic pocket where it is proposed to make a crucial interaction with the conserved acid residue that caps the groove ${ }^{55}$.

What type of protein substrates might PDIP38 recognise? One possibility, that would be specific to the matrix compartment, is the recognition of incompletely or aberrantly processed matrix proteins, which retain their $\mathrm{N}$-terminal presequence. These presequences are generally enriched in hydrophobic and basic residues, hence we speculate that PDIP38 might be responsible for the recognition these incorrectly processed or unprocessed matrix proteins, delivering them to CLPXP for removal. This role is somewhat similar to that of the E. coli ClpX adaptor protein - SspB (which is responsible for the recognition of incomplete translation products that bear a largely hydrophobic C-terminal recognition motif known as the SsrA-tag ${ }^{41,56,57}$. An alternate possibility involves the more general recognition of exposed hydrophobic patches that are found in misfolded proteins that accumulate in response proteostatic stress. Conversely, PDIP38 may be required for the conditional recognition of proteins that expose hydrophobic motifs at either their $\mathrm{N}$ - or C-termini.

In summary, we show that PDIP38 is a novel component of the proteostasis network in mammalian mitochondria. Not only does PDIP38 modulate CLPXP substrate specificity and inhibit the LONMmediated turnover of CLPX in vitro, but it also stabilises the steady state levels of CLPX in vivo. As such we propose that PDIP38 represents a novel mitochondrial adaptor protein for the CLPXP protease. Consistently, the atomic structure of PDIP38 revealed that the protein is composed of two domains separated by an $\alpha$-helical hinge. The $\mathrm{N}$-terminal YccV-like domain is crucial for interaction with the adaptor binding loop within the ZBD of CLPX, while the C-terminal domain contains a conserved hydrophobic groove which is proposed to facilitate substrate binding and hence delivery to CLPX. Significantly, the residues that line this hydrophobic groove are highly conserved across DUF525 containing proteins, from bacteria to humans. Hence, we speculate that the bacterial PDIP38 homolog (i.e. ApaG) may also play a role in protein turnover. An important challenge for the future will be the in vivo dissection of this system to identify the physiological substrates of the CLPXP protease that are delivered by PDIP38.

\section{ACKNOWLEDGEMENTS}

This work was supported by an Australian Research Council (ARC) Discovery Project (DP0770013) to D.A.D and K.N.T., and ARC Future Fellowship to K.N.T. (FT0992033) and an ARC Australian Research Fellowship to D.A.D (DP110103936). P.R.S. and H.Z were supported by a La Trobe University Postgraduate Award, E.J.B. was supported by Australian Postgraduate Awards and T.S. 


\section{3-file00.docx}

was supported Cooperative Research Centre postgraduate award. We thank Mia Miasari for cloning of PDIP38 $8_{N}$ and PDIP38 ${ }_{C}$ into pGEX-4T.

\section{Author contribution:}

Conceptualization, D.A.D. and K.N.T.; Methodology, D.A.D., K.N.T. and K.Z.; Investigation, P.R.S., E.J.B., H.Z., V.J.S., L.J.V., T.S. and K.Z.; Writing - Original Draft, D.A.D., K.N.T. and K.Z.; Supervision, Project Administration and Funding Acquisition, D.A.D. and K.N.T.

\section{Declaration of interests:}

The authors declare no competing interests.

\section{References}

1 Liu, L., Rodriguez-Belmonte, E. M., Mazloum, N., Xie, B. \& Lee, M. Y. Identification of a novel protein, PDIP38, that interacts with the p50 subunit of DNA polymerase delta and proliferating cell nuclear antigen. $J$ Biol Chem 278, 10041-10047, doi:10.1074/jbc.M208694200 (2003).

2 Klaile, E., Kukalev, A., Obrink, B. \& Muller, M. M. PDIP38 is a novel mitotic spindleassociated protein that affects spindle organization and chromosome segregation. Cell Cycle 7, 3180-3186, doi:10.4161/cc.7.20.6813 (2008).

3 Tissier, A. et al. Crosstalk between replicative and translesional DNA polymerases: PDIP38 interacts directly with Poleta. DNA Repair (Amst) 9, 922-928, doi:10.1016/j.dnarep.2010.04.010 (2010).

4 Wong, A. et al. PDIP38 is translocated to the spliceosomes/nuclear speckles in response to UV-induced DNA damage and is required for UV-induced alternative splicing of MDM2. Cell Cycle 12, 3184-3193, doi:10.4161/cc.26221 (2013).

5 Xie, B. et al. Further characterization of human DNA polymerase delta interacting protein 38. J Biol Chem 280, 22375-22384, doi:10.1074/jbc.M414597200 (2005).

6 Klaile, E. et al. The cell adhesion receptor carcinoembryonic antigen-related cell adhesion molecule 1 regulates nucleocytoplasmic trafficking of DNA polymerase delta-interacting protein 38. J Biol Chem 282, 26629-26640, doi:10.1074/jbc.M701807200 (2007).

7 Arakaki, N. et al. Regulation of mitochondrial morphology and cell survival by Mitogenin I and mitochondrial single-stranded DNA binding protein. Biochim Biophys Acta 1760, 13641372, doi:10.1016/j.bbagen.2006.05.012 (2006).

8 Sutliff, R. L. et al. Polymerase delta interacting protein 2 sustains vascular structure and function. Arterioscler Thromb Vasc Biol 33, 2154-2161, doi:10.1161/ATVBAHA.113.301913 (2013).

9 Lyle, A. N. et al. Poldip2, a novel regulator of Nox4 and cytoskeletal integrity in vascular smooth muscle cells. Circ Res 105, 249-259, doi:10.1161/CIRCRESAHA.109.193722 (2009). 


\section{3-file00.docx}

42810 Kim, Y. et al. Essential role of POLDIP2 in Tau aggregation and neurotoxicity via autophagy/proteasome inhibition. Biochem Biophys Res Commun 462, 112-118, doi:10.1016/j.bbrc.2015.04.084 (2015).

11 Grinchuk, O. V., Motakis, E. \& Kuznetsov, V. A. Complex sense-antisense architecture of TNFAIP1/POLDIP2 on 17q11.2 represents a novel transcriptional structural-functional gene module involved in breast cancer progression. BMC Genomics 11 Suppl 1, S9, doi:10.1186/1471-2164-11-S1-S9 (2010).

12 Lowth, B. R. et al. Substrate recognition and processing by a Walker B mutant of the human mitochondrial AAA+ protein CLPX. J Struct Biol 179, 193-201, doi:10.1016/j.jsb.2012.06.001 (2012).

13 Cheng, X. et al. PDIP38 associates with proteins constituting the mitochondrial DNA nucleoid. J Biochem 138, 673-678, doi:10.1093/jb/mvi169 (2005).

14 Paredes, F. et al. Poldip2 is an oxygen-sensitive protein that controls PDH and alphaKGDH lipoylation and activation to support metabolic adaptation in hypoxia and cancer. Proc Natl Acad Sci U S A 115, 1789-1794, doi:10.1073/pnas.1720693115 (2018).

15 Sala, A. J., Bott, L. C. \& Morimoto, R. I. Shaping proteostasis at the cellular, tissue, and organismal level. J Cell Biol 216, 1231-1241, doi:10.1083/jcb.201612111 (2017).

16 Kirstein, J., Moliere, N., Dougan, D. A. \& Turgay, K. Adapting the machine: adaptor proteins for Hsp100/Clp and AAA+ proteases. Nat Rev Microbiol 7, 589-599, doi:10.1038/nrmicro2185 (2009).

17 Nishimura, K. \& van Wijk, K. J. Organization, function and substrates of the essential Clp protease system in plastids. Biochim Biophys Acta 1847, 915-930, doi:10.1016/j.bbabio.2014.11.012 (2015).

18 Varshavsky, A. The N-end rule pathway and regulation by proteolysis. Protein Sci 20, 12981345, doi:10.1002/pro.666 (2011).

19 Gur, E., Ottofueling, R. \& Dougan, D. A. Machines of destruction - AAA+ proteases and the adaptors that control them. Subcell Biochem 66, 3-33, doi:10.1007/978-94-007-5940-4_1 (2013).

20 Olivares, A. O., Baker, T. A. \& Sauer, R. T. Mechanical Protein Unfolding and Degradation. Annu Rev Physiol 80, 413-429, doi:10.1146/annurev-physiol-021317-121303 (2018).

21 Striebel, F., Kress, W. \& Weber-Ban, E. Controlled destruction: AAA+ ATPases in protein degradation from bacteria to eukaryotes. Curr Opin Struct Biol 19, 209-217, doi:10.1016/j.sbi.2009.02.006 (2009).

22 Yu, H. \& Matouschek, A. Recognition of Client Proteins by the Proteasome. Annu Rev Biophys 46, 149-173, doi:10.1146/annurev-biophys-070816-033719 (2017).

23 Glynn, S. E. Multifunctional Mitochondrial AAA Proteases. Front Mol Biosci 4, 34, doi:10.3389/fmolb.2017.00034 (2017).

24 Bezawork-Geleta, A., Brodie, E. J., Dougan, D. A. \& Truscott, K. N. LON is the master protease that protects against protein aggregation in human mitochondria through direct degradation of misfolded proteins. Sci Rep 5, 17397, doi:10.1038/srep17397 (2015). 


\section{3-file00.docx}

46825 Bota, D. A. \& Davies, K. J. Lon protease preferentially degrades oxidized mitochondrial aconitase by an ATP-stimulated mechanism. Nat Cell Biol 4, 674-680, doi:10.1038/ncb836 (2002).

47126 Fukuda, R. et al. HIF-1 regulates cytochrome oxidase subunits to optimize efficiency of respiration in hypoxic cells. Cell 129, 111-122, doi:10.1016/j.cell.2007.01.047 (2007).

27 Tian, Q. et al. Lon peptidase 1 (LONP1)-dependent breakdown of mitochondrial 5aminolevulinic acid synthase protein by heme in human liver cells. J Biol Chem 286, 2642426430, doi:10.1074/jbc.M110.215772 (2011).

28 Kubota, Y. et al. Novel Mechanisms for Heme-dependent Degradation of ALAS1 Protein as a Component of Negative Feedback Regulation of Heme Biosynthesis. J Biol Chem 291, 20516-20529, doi:10.1074/jbc.M116.719161 (2016).

29 Yien, Y. Y. et al. Mutation in human CLPX elevates levels of delta-aminolevulinate synthase and protoporphyrin IX to promote erythropoietic protoporphyria. Proc Natl Acad Sci U S A 114, E8045-E8052, doi:10.1073/pnas.1700632114 (2017).

30 Kardon, J. R. et al. Mitochondrial ClpX Activates a Key Enzyme for Heme Biosynthesis and Erythropoiesis. Cell 161, 858-867, doi:10.1016/j.cell.2015.04.017 (2015).

31 Szczepanowska, K. et al. CLPP coordinates mitoribosomal assembly through the regulation of ERAL1 levels. EMBO J 35, 2566-2583, doi:10.15252/embj.201694253 (2016).

32 Pryde, K. R., Taanman, J. W. \& Schapira, A. H. A LON-ClpP Proteolytic Axis Degrades Complex I to Extinguish ROS Production in Depolarized Mitochondria. Cell Rep 17, 25222531, doi:10.1016/j.celrep.2016.11.027 (2016).

33 Brodie, E. J., Zhan, H., Saiyed, T., Truscott, K. N. \& Dougan, D. A. Perrault syndrome type 30311-1 (2018).

Jenkinson, E. M. et al. Perrault syndrome is caused by recessive mutations in CLPP, encoding a mitochondrial ATP-dependent chambered protease. Am J Hum Genet 92, 605613, doi:10.1016/j.ajhg.2013.02.013 (2013).

Gispert, S. et al. Loss of mitochondrial peptidase Clpp leads to infertility, hearing loss plus growth retardation via accumulation of CLPX, mtDNA and inflammatory factors. Hum Mol Genet 22, 4871-4887, doi:10.1093/hmg/ddt338 (2013).

Ishizawa, J. et al. Mitochondrial ClpP-Mediated Proteolysis Induces Selective Cancer Cell Lethality. Cancer Cell 35, 721-737 e729, doi:10.1016/j.ccell.2019.03.014 (2019).

50037 Wang, S. \& Dougan, D. A. The Direct Molecular Target for Imipridone ONC201 Is Finally Established. Cancer Cell 35, 707-708, doi:10.1016/j.ccell.2019.04.010 (2019).

Nishimura, K. et al. Discovery of a Unique Clp Component, ClpF, in Chloroplasts: A Delivery. Plant Cell 27, 2677-2691, doi:10.1105/tpc.15.00574 (2015).

Yeom, J. \& Groisman, E. A. Activator of one protease transforms into inhibitor of another in response to nutritional signals. Genes Dev, doi:10.1101/gad.325241.119 (2019). 


\section{3-file00.docx}

Dougan, D. A., Reid, B. G., Horwich, A. L. \& Bukau, B. ClpS, a substrate modulator of the ClpAP machine. Mol Cell 9, 673-683 (2002).

Dougan, D. A., Weber-Ban, E. \& Bukau, B. Targeted delivery of an ssrA-tagged substrate by the adaptor protein SspB to its cognate AAA+ protein ClpX. Mol Cell 12, 373-380 (2003).

42 Wojtyra, U. A., Thibault, G., Tuite, A. \& Houry, W. A. The N-terminal zinc binding domain of $\mathrm{ClpX}$ is a dimerization domain that modulates the chaperone function. J Biol Chem $\mathbf{2 7 8}$, 48981-48990 (2003).

Truscott, K. N., Lowth, B. R., Strack, P. R. \& Dougan, D. A. Diverse functions of mitochondrial AAA+ proteins: protein activation, disaggregation, and degradation. Biochem Cell Biol 88, 97-108, doi:10.1139/o09-167 (2010).

Mitchell, A. L. et al. InterPro in 2019: improving coverage, classification and access to protein sequence annotations. Nucleic Acids Res 47, D351-D360, doi:10.1093/nar/gky1100 (2019).

Watanabe, K., Yumimoto, K. \& Nakayama, K. I. FBXO21 mediates the ubiquitylation and proteasomal degradation of EID1. Genes Cells 20, 667-674, doi:10.1111/gtc.12260 (2015).

Zhang, C. et al. Peptidic degron in EID1 is recognized by an SCF E3 ligase complex containing the orphan F-box protein FBXO21. Proc Natl Acad Sci U S A 112, 15372-15377, doi:10.1073/pnas.1522006112 (2015).

Montandon, C., Dougan, D. A. \& van Wijk, K. J. N-degron specificity of chloroplast ClpS1 in plants. FEBS Lett 593, 962-970, doi:10.1002/1873-3468.13378 (2019).

Erbse, A. et al. ClpS is an essential component of the N-end rule pathway in Escherichia coli. Nature 439, 753-756, doi:10.1038/nature04412 (2006).

Puri, N. \& Karzai, A. W. HspQ Functions as a Unique Specificity-Enhancing Factor for the AAA+ Lon Protease. Mol Cell 66, 672-683 e674, doi:10.1016/j.molcel.2017.05.016 (2017).

Varshavsky, A. N-degron and C-degron pathways of protein degradation. Proc Natl Acad Sci U S A 116, 358-366, doi:10.1073/pnas.1816596116 (2019). system for high-level expression and easy purification of authentic recombinant proteins. Protein Sci 13, 1331-1339, doi:10.1110/ps.04618904 (2004).

Kang, S. G. et al. Functional proteolytic complexes of the human mitochondrial ATPdependent protease, hClpXP. J Biol Chem 277, 21095-21102, doi:10.1074/jbc.M201642200 (2002). 2101, doi:10.1111/febs.13721 (2016). mediated degradation. Mol Cell Biol 28, 7126-7138, doi:10.1128/MCB.00897-08 (2008).

54455 Mallampalli, R. K. et al. Targeting $\mathrm{F}$ box protein Fbxo3 to control cytokine-driven inflammation. J Immunol 191, 5247-5255, doi:10.4049/jimmunol.1300456 (2013). 


\section{3-file00.docx}

$54656 \quad$ Levchenko, I., Grant, R. A., Wah, D. A., Sauer, R. T. \& Baker, T. A. Structure of a delivery protein for an AAA+ protease in complex with a peptide degradation tag. Mol Cell 12, 365$372(2003)$.

57 Levchenko, I., Seidel, M., Sauer, R. T. \& Baker, T. A. A specificity-enhancing factor for the ClpXP degradation machine. Science 289, 2354-2356 (2000).

58 Park, E. Y. et al. Structural basis of SspB-tail recognition by the zinc binding domain of ClpX. J Mol Biol 367, 514-526, doi:10.1016/j.jmb.2007.01.003 (2007).

59 Truscott, K. N. et al. A presequence- and voltage-sensitive channel of the mitochondrial preprotein translocase formed by Tim23. Nat Struct Biol 8, 1074-1082 (2001).

60 Zheng, L., Baumann, U. \& Reymond, J. L. An efficient one-step site-directed and sitesaturation mutagenesis protocol. Nucleic Acids Res 32, e115, doi:10.1093/nar/gnh110 (2004).

61 Ninnis, R. L., Spall, S. K., Talbo, G. H., Truscott, K. N. \& Dougan, D. A. Modification of PATase by L/F-transferase generates a ClpS-dependent $\mathrm{N}$-end rule substrate in Escherichia coli. EMBO J 28, 1732-1744, doi:10.1038/emboj.2009.134 (2009).

62 Schagger, H. \& von Jagow, G. Tricine-sodium dodecyl sulfate-polyacrylamide gel electrophoresis for the separation of proteins in the range from 1 to $100 \mathrm{kDa}$. Anal Biochem 166, 368-379 (1987).

63 Geissler, A. et al. The mitochondrial presequence translocase: an essential role of Tim50 in directing preproteins to the import channel. Cell 111, 507-518 (2002).

64 Bezawork-Geleta, A., Saiyed, T., Dougan, D. A. \& Truscott, K. N. Mitochondrial matrix proteostasis is linked to hereditary paraganglioma: LON-mediated turnover of the human flavinylation factor SDH5 is regulated by its interaction with SDHA. FASEB J 28, 17941804, doi:10.1096/fj.13-242420 (2014).

65 Stojanovski, D., Pfanner, N. \& Wiedemann, N. Import of proteins into mitochondria. Methods Cell Biol 80, 783-806, doi:10.1016/S0091-679X(06)80036-1 (2007).

66 Kabsch, W. Integration, scaling, space-group assignment and post-refinement. Acta Crystallographica Section D 66, 133-144, doi:doi:10.1107/S0907444909047374 (2010).

67 Kabsch, W. XDS. Acta Crystallographica Section D $\quad$ 66, 125-132, doi:doi:10.1107/S0907444909047337 (2010).

68 Afonine, P. V. et al. Joint X-ray and neutron refinement with phenix.refine. Acta Crystallogr D Biol Crystallogr 66, 1153-1163, doi:10.1107/S0907444910026582 (2010).

\section{Figure Legends}

\section{Figure 1. Human PDIP38 is imported into mitochondrial where it interacts with CLPX.}

a. Import of $\left[{ }^{35} \mathrm{~S}\right]$-labelled PDIP38 preprotein into mitochondria isolated from HeLa cells, in the presence or absence of a membrane potential $(\Delta \psi)$ as indicated, treated with (lanes $7-12$ ) or without (lanes 1 -6) proteinase K (Prot. K). Samples were separated by $15 \%$ Tris-glycine SDSPAGE and analysed by digital autoradiography. b. Mitochondria were incubated, either in an 


\section{3-file00.docx}

584

585

586

587

588

589

590

591

592

593

594

595

596

597

598

599

600

601

602

603

604

605

606

607

608

609

610

611

612

613

614

615

616

617

osmotic buffer (lanes $1-4$ ), isotonic buffer (Swelling) to rupture the outer membrane (lanes $5-8$ ) or buffer containing Triton X-100 (lanes 9 - 12), in the absence (lanes 1, 5 and 9) or presence (lanes $2-4,6-8$ and $10-12$ ) of Prot. K for the indicated time. Samples were separated by $15 \%$ Trisglycine SDS-PAGE and subjected to immunoblotting with the appropriate antisera to visualize endogenous proteins. *, non-specific cross-reactive protein in PDIP38 antisera c. Specific immunoprecipitation of endogenous PDIP38 from detergent solubilized mitochondria using antiPDIP38 polyclonal antibodies showing co-immunoprecipitation of endogenous CLPX. The input represents $50 \%$ of total mitochondrial lysate subjected to IP. d. Specific immunoprecipitation of endogenous CLPX from detergent solubilized mitochondria using anti-CLPX polyclonal antibodies showing co-immunoprecipitation of endogenous PDIP38. The input represents $50 \%$ of total mitochondrial lysate subjected to IP.

\section{Figure 2. The interaction between PDIP38 and CLPX is mediated by the ZBD of CLPX. a.} Cartoon representation of human CLPX domain structure. b. Coomassie stained $16.5 \%$ Tricinebuffered SDS-PAGE of PDIP38 pull-down from E. coli lysate expressing recombinant GST-PDIP38 using bead immobilised $\mathrm{H}_{10}$ CLPX (CLPX) relative to beads only (control). T, total E. coli lysate with expressed GST-PDIP38; U, unbound fraction; E, eluted fraction. c. Coomassie-stained 16.5\% Tricine-buffered SDS-PAGE of PDIP38 pull-down from E. coli lysate expressing recombinant GST-PDIP38 using bead immobilised $\mathrm{H}_{10}$ CLPX E-domain (CLPX $\mathrm{E}_{\mathrm{E}}$ ) and $\mathrm{H}_{10}$ CLPX Zinc binding domain $\left(\mathrm{CLPX}_{\mathrm{ZBD}}\right)$ relative to beads only (control). T, total lysate expressing GST-PDIP38; U, unbound fraction; E, eluted fraction. For comparison purified recombinant CLPX $_{\mathrm{E}}$ (lane 11), CLPX $_{\mathrm{ZBD}}$ (lane 12) and GST-PDIP38 (lane 13) are shown. d. Complex formation of PDIP38 and CLPX $_{\mathrm{ZBD}}$ was monitored by size exclusion chromatography (SEC) using a Superose 12 column. Elution profiles of PDIP38 (top panel), CLPX $\mathrm{ZBD}$ (middle panel) or PDIP38 in the presence of $\mathrm{CLPX}_{\mathrm{ZBD}}$ (bottom panel) were measured at $280 \mathrm{~nm}\left(\mathrm{~A}_{280}\right)$. Arrows indicate the peak elution volume of Albumin (67 kDa), Ovalbumin (43 kDa), chymotrypsin A (25 kDa) and Ribonuclease (13.7 kDa).

\section{Figure 3. The N-terminal domain of PDIP38 interacts with CLPX.}

a. Limited proteolysis of native His 10 -tagged PDIP38 using thermolysin. Samples were analyzed by Coomassie stained 16.5\% Tricine-buffered SDS-PAGE. *, thermolysin. f1, f1', f2, f2', fragments of PDIP38. b. Schematic representation of GST-PDIP38 fusion constructs. Preprotein numbering is used. c. In vitro pull-down using Ni-NTA agarose with (lanes 2, 4 and 6) or without (lanes 3, 5 and 7) purified immobilised $\mathrm{H}_{10} \mathrm{CLPX}$, incubated with E. coli lysate expressing GST-PDIP38 (lanes 2 3), GST-PDIP38 $8_{N}$ (lanes $4-5$ ) or GST-PDIP38 $8_{C}$ (lanes $6-7$ ). Eluted fractions are shown with 
40922693-file00.docx

618 samples analyzed by Coomassie Brilliant Blue (CBB) staining or immunoblotting (with anti-GST)

619 following separation by SDS-PAGE. As a control, purified $\mathrm{H}_{10}$ CLPX (lane1) is shown.

621 Figure 4. PDIP38 inhibits the CLPXP-mediated degradation of a model substrate via adaptor-

\section{2 like docking to CLPX $X_{Z B D}$}

623 a. In vitro degradation of FITC-labelled casein by CLPXP protease in the absence and presence of

$6242.4 \mu \mathrm{M}$ untagged PDIP38. Samples were separated by SDS-PAGE and analysed by fluorescence 625 detection (FITC-casein) then CBB staining (PDIP38). b. In vitro pull-down, in which purified 626 human (lanes 1 - 2) or E. coli (lanes 3 - 4) ZBD was immobilised to Ni-NTA agarose beads and 627 incubated with (lanes 1 and 3) or without (lanes 2 and 4) an E. coli lysate expressing GST-PDIP38. 628 Eluted fractions are shown with samples analysed by Coomassie Brilliant Blue (CBB) staining. c. 629 Amino acid sequence alignment of eukaryotic and prokaryotic CLPX homologs showing the ZBD 630 only. The adaptor binding loop in prokaryotic ClpX homologs is highlighted in the boxed section. 631 ClpX sequences are Homo sapiens (O76031); Mus musculus (Q9JHS4); Bovine (F1N155); Danio 632 rerio (Q66HW5), Drosophila melanogaster (Q9VDS7), Escherichia coli (P0A6H1), Caulobacter 633 crescentus (P0CAU2), Mycobacterium tuberculosis (P9WPB9) and Bacillus subtilus (P50866). $d$. 634 Ribbon diagram of E. coli CLPX $_{\mathrm{ZBD}}$ (PDB: $2 \mathrm{DS} 6^{58}$, blue) overlaid with a model of the human $635 \mathrm{CLPX}_{\mathrm{ZBD}}$ (red) highlighting the position of the putative adaptor-binding loop in human CLPX 636 (circled). The space-filling model of E. coli $\mathrm{ClpX} \mathrm{N-domain} \mathrm{is} \mathrm{also} \mathrm{shown} \mathrm{in} \mathrm{light} \mathrm{blue.} \mathrm{e.} \mathrm{In} \mathrm{vitro}$ 637 pull-down, in which purified wild type (lanes 1 - 2) or mutant (lanes 3 - 4) ZBD and wild type 638 (lanes 5 - 6) or mutant (lanes 7 - 8) human $\mathrm{H}_{10}$ CLPX was immobilised to Ni-NTA agarose beads 639 and incubated with (lanes 1, 3, 5 and 7) or without (lanes 2, 4 and 6) an E. coli lysate expressing 640 untagged PDIP38. Eluted proteins were separated by SDS-PAGE and visualized by Coomassie 641 Brilliant Blue (CBB) staining.

642 Figure 5. PDIP38 stabilises CLPX protecting it from LONM-mediated degradation.

644 a. Representative immunoblots illustrating the steady state levels of CLPX (upper gel strip), 645 PDIP38 (middle gel strip) and CLPP (lower gel strip) in PDIP38-depleted HeLa cells (lanes 1 - 3) 646 relative to control HeLa cells (lanes $4-6$ ). Samples were collected at the indicated times post647 transfection of either Silencer Select siRNA (22994) targeting PDIP38 or a negative control siRNA 648 (control). Proteins were separated by 16.5\% Tris-Tricine SDS-PAGE. *, non-specific cross-reactive 649 protein in PDIP38 antisera. The lower panel shows the quantitation of CLPX levels from three 650 independent experiments, in PDIP38 depleted HeLa cells (grey bars) in comparison a negative 651 control siRNA (white bars). Error bars represent the standard error of the mean (S.E.M.) of at least 


\section{3-file00.docx}

652 three independent experiments. b. In vitro degradation of CLPX by LONM 6 protease (400 $\mathrm{nM})$ in 653 the absence or presence of $1 \mu \mathrm{M}$ PDIP38. Samples were separated by $10 \%$ Tris-Tricine SDS-PAGE 654 and analysed by CBB staining. * LONM impurity. c. Quantitation of in vitro degradation of CLPX 655 by $\mathrm{LONM}_{6}(400 \mathrm{nM})$ in the absence (open symbols) or presence (closed symbols) of untagged 656 PDIP38. Samples were separated by SDS-PAGE and analysed by CBB staining. Error bars 657 represent the S.E.M. of three independent experiments.

658

Figure 6 Structure of PDIP38 highlighting the conserved hydrophobic groove

660 a. Ribbon representation of human PDIP38 highlighting its three domains. The N-terminal yccV661 like domain (green) and the C-terminal DUF525 domain (blue) are separated by a hinge or linker 662 domain (magenta). b. Secondary elements are illustrated above the amino acid sequence. Red 663 scissors indicate the site of cleavage by thermolysin. Underlined protein sequence represents 664 disordered regions of the protein structure. c. Ribbon representation of human PDIP38, highlighting 665 the conserved hydrophobic residues that line the binding groove. d-f. Hydrophobic surface 666 representation of (d) PDIP38 illustrating the substrate binding groove compared to (e) Human Fbx3 667 DUF525 domain (PDB code 5HDW) and (f) Xanthomonas axonopodis ApaG (PDB code 2F1E). 668 All figures were generated in ChimeraX_Daily.

\section{Methods}

\section{$671 \quad$ Plasmids}

672 For in vitro transcription and translation of human PDIP38, pOTB7/PDIP38 was obtained from the 673 I.M.A.G.E. Consortium (ID 3349399). For the heterologous expression of PDIP38 in E. coli, the 674 cDNA coding for mature PDIP38 (residues 52-368) was amplified by PCR from pOTB7/PDIP38 675 using the appropriate primers (Supplementary Table 3) and cloned into either pHUE ${ }^{51}$ between Sac 676 II and Hind III (to express untagged PDIP38), pET10N ${ }^{59}$ between Not I and Xho I (to express 677 PDIP38 with an N-terminal $\mathrm{H}_{10}$ tag), pET10C ${ }^{59}$ between Nde I and Not I (to express PDIP38 as a 678 C-terminal $\mathrm{H}_{10}$ fusion protein), or pGEX-4T-1 between Bam $\mathrm{HI}$ and Xho I (to express PDIP38 as an 679 N-terminal Glutathione S-transferase (GST) fusion protein). To generate PDIP38 (residues 52-153) 680 and PDIP38 ${ }_{\mathrm{C}}$ (residues 157-368) fused to GST, pGEX-4T/PDIP38 was subjected to site-directed 681 mutagenesis ${ }^{60}$ using primers PDIP_bam1 and PDIP_bam2 (see Supplementary Table 3). The 682 resulting plasmid (pDT1367, see Supplementary Table 4) contained a stop codon and an additional 683 Bam HI site (and was used directly for expression of GST-PDIP38 $8_{\mathrm{N}}$ ). To generate GST-PDIP38 $8_{\mathrm{C}}$, 684 pDT1367 was digested with Bam HI, the cut vector ligated lacking the PDIP3 $8_{N}$ fragment to 685 generate pDT1362. Plasmids for bacterial expression of human CLPX (full-length and domain 


\section{3-file00.docx}

686 mutants) and human CLPP (either His-tagged and untagged) were described previously ${ }^{33}$. For

687 expression of $\mathrm{CLPX}_{4 \mathrm{~A}}$ and $\mathrm{ZBD}_{4 \mathrm{~A}}, \mathrm{pET} 10 \mathrm{C} / h C L P X_{4 \mathrm{~A}}$ and $\mathrm{pET} 10 \mathrm{C} / h Z B D_{4 \mathrm{~A}}$ were generated by site

688 directed mutagenesis using appropriate primers (see Supplementary Table 3). For details of primer

689 sequences and plasmid constructs, refer to Supplementary information. All clones were confirmed

690 by Sanger sequencing.

\section{Proteins}

692 Recombinant proteins were expressed, either in BL21-CodonPlus®(DE3)-RIL or XL1-Blue 693 (Agilent) Escherichia coli cells, as appropriate. His-tagged $\left(\mathrm{H}_{6^{-}}\right.$or $\left.\mathrm{H}_{10^{-}}\right)$recombinant proteins were 694 purified from clarified E. coli lysates under native conditions by immobilised metal affinity 695 chromatography using Ni-NTA agarose (Qiagen) essentially as described ${ }^{12}$ using $50 \mathrm{mM}$ Tris-HCl, 696 [pH 8.0], $300 \mathrm{mM} \mathrm{NaCl}$ supplemented with an appropriate concentration of imidazole for binding 697 (10 or $20 \mathrm{mM})$, washing (20 mM or $65 \mathrm{mM})$ and elution $(250 \mathrm{mM}$ or $500 \mathrm{mM})$. Purified His $6_{6}-\mathrm{Ub}-$ 698 PDIP38 and His $_{6}$-Ub-CLPP were cleaved using His $_{6}$-Usp2cc ${ }^{51}$ and the untagged mature proteins 699 recovered via a method outlined previously ${ }^{51,61}$. GST-PDIP38 was purified by affinity 700 chromatography using GSH agarose (Bioserve) as outlined by the manufacturer. Radiolabelled 701 PDIP38 preprotein was synthesized using TNT® SP6 Quick Coupled Transcription-Translation 702 System (Promega) with undigested pOTB7/PDIP38 as template and $11 \mu \mathrm{Ci}$ of $703\left[{ }^{35} \mathrm{~S}\right]$ Met/CysEXPRE ${ }^{35} \mathrm{~S}^{35} \mathrm{~S}$ protein labelling mix (specific activity of $>1000 \mathrm{Ci} / \mathrm{mmol}$ ) from Perkin 704 Elmer. Protein Assay (Bio-Rad) was used to determine protein concentrations using bovine serum 705 albumin (Thermo Scientific) as a standard. Protein concentrations refer to the protomer unless 706 otherwise stated. FITC-casein, thermolysin, proteinase $\mathrm{K}$ and lysozyme were purchased from 707 Sigma-Aldrich, DNase I was purchased from Gold Biotechnology. SeeBlue ${ }^{\circ}$ Plus2 pre-stained and 708 Mark12 ${ }^{\mathrm{TM}}$ unstained protein standards were from Life Technologies.

\section{Electrophoresis and protein detection}

710 Proteins were separated using either glycine- or Tricine-buffered ${ }^{62}$ SDS-PAGE. Protein samples in 7111 x SDS-PAGE sample buffer (80 mM Tris-HCl [pH 6.8], 2\% (w/v) SDS, 5\% (v/v) glycerol, 100 $712 \mathrm{mM}$ DTT and $0.02 \%(\mathrm{w} / \mathrm{v})$ bromophenol blue) were heat treated at $95{ }^{\circ} \mathrm{C}$ for 5 min before 713 separation. For visualization of proteins, gels were stained with Coomassie Brilliant Blue R250 714 solution (CBB) or transferred to polyvinyldiflouride (PVDF) membrane using semi-dry method for 715 immunoblotting. Primary antibodies used were anti-PDIP38 (POLDIP2; Abcam), anti-PDIP38 716 (125/88; generated in rabbit using purified recombinant PDIP38- $\mathrm{H}_{10}$ as antigen), affinity purified 717 anti-CLPX ${ }^{12}$, anti-LONM ${ }^{12}$, anti-TIM23 (BD Biosciences), anti-SDHA (Invitrogen), anti-GST 718 (GE Healthcare), anti-GAPDH (Life Technologies) and anti-mtHSP60 (N. Hoogenraad, La Trobe 719 University). Peroxidase coupled secondary antibodies were anti-rabbit, anti-mouse and anti-goat 
40922693-file00.docx

720 IgG (Sigma-Aldrich). Antibody complexes were detected using enhanced chemiluminescence 721 detection reagents (GE Healthcare) and digital images captured using GeneSnap (SynGene) or 722 Image Lab ${ }^{\mathrm{TM}}$ (Bio-Rad) Software. FITC-casein was detected by in gel fluorescence (excitation 488 $723 \mathrm{~nm}$ and emission $526 \mathrm{~nm}$ ) while radiolabelled proteins were detected by exposing dried gels to 724 phosphor screens. Imaging was performed using a Typhoon ${ }^{\mathrm{TM}}$ Trio variable mode imager and 725 analysed using ImageQuant software (GE Healthcare).

\section{Limited proteolysis}

$727 \mathrm{H}_{10}$ PDIP38 $(0.1 \mathrm{mg} / \mathrm{ml})$ was subjected to limited proteolysis using thermolysin $(0.01 \mathrm{mg} / \mathrm{ml})$ at 30 $728{ }^{\circ} \mathrm{C}$ in $50 \mathrm{mM}$ Tris- $\mathrm{HCl}[\mathrm{pH} 7.0], 150 \mathrm{mM} \mathrm{NaCl}$ and $5 \mathrm{mM} \mathrm{CaCl}_{2}$. To terminate the reaction, 729 samples were treated with $2 \mathrm{mM}$ PMSF and heated at $95^{\circ} \mathrm{C}$ in $1 \mathrm{x}$ SDS-PAGE sample buffer.

\section{Degradation assays}

731 The CLPXP-mediated degradation of FITC-casein was performed essentially as described ${ }^{52}$. 732 Briefly, $0.4 \mu \mathrm{M} \mathrm{CLPX}{ }_{6} \mathrm{P}_{14}$ was preincubated (at $30{ }^{\circ} \mathrm{C}$ for $5 \mathrm{~min}$ ) in proteolysis buffer (50 $\mathrm{mM}$ Tris$733 \mathrm{HCl}[\mathrm{pH} 8.0], 100 \mathrm{mM} \mathrm{KCl}, 20 \mathrm{mM} \mathrm{MgCl} 2,1 \mathrm{mM}$ DTT, $0.02 \%$ (v/v) Triton X-100, $10 \%$ (v/v) 734 glycerol) with FITC-casein $(0.3 \mu \mathrm{M})$ in the absence or presence of $2.4 \mu \mathrm{M}$ untagged PDIP38. To 735 initiate degradation $5 \mathrm{mM}$ ATP was added and samples were incubated at $30{ }^{\circ} \mathrm{C}$ for the times 736 indicated. Reactions were terminated by the addition of $1 \mathrm{x}$ sample buffer and the proteins 737 denatured at $95^{\circ} \mathrm{C}$ for $5 \mathrm{~min}$.

\section{In vitro binding analysis}

739 The in vitro binding analysis was adapted from the method outlined in ${ }^{63}$. E. coli cells containing 740 expressed GST-PDIP38, GST-PDIP38 ${ }_{\mathrm{N}}$, GST-PDIP38 $\mathrm{C}$ or untagged PDIP38, were resuspended (5 $741 \mathrm{ml} / \mathrm{g}$ wet weight of cells) in Binding Buffer (20 mM HEPES-KOH [pH 7.5], $100 \mathrm{mM} \mathrm{K}(\mathrm{OAc}), 10$ $742 \mathrm{mM} \mathrm{Mg}(\mathrm{OAc}), 10 \%(\mathrm{v} / \mathrm{v})$ glycerol, $65 \mathrm{mM}$ imidazole) supplemented with $0.5 \%(\mathrm{v} / \mathrm{v})$ Triton X743 100, EDTA free protease inhibitor cocktail (Roche), $2 \mathrm{mM}$ PMSF and DNase I (10 $\mu \mathrm{g} / \mathrm{ml})$ then 744 subjected to chemical lysis with lysozyme $(0.2 \mathrm{mg} / \mathrm{ml})$. Cell free lysates or purified untagged 745 PDIP38, as appropriate, were applied to Ni-NTA agarose beads either lacking or containing 746 immobilised $\mathrm{H}_{10}$-tagged CLPX, CLPX $\mathrm{ZBD}, \mathrm{CLPX}_{\mathrm{E}}$, ecClpX $\mathrm{ZBD}, \mathrm{CLPX}_{4 \mathrm{~A}}$ or $\mathrm{ZBD}_{4 \mathrm{~A}}$ and incubated 747 with end-over-end mixing at $4{ }^{\circ} \mathrm{C}$ for $30 \mathrm{~min}$. The beads were then washed with 5 bed volumes (BV) 748 of Binding Buffer supplemented with $0.5 \%$ (v/v) Triton X-100 followed by 10 BV of Wash Buffer 749 (Binding buffer supplemented with $0.25 \%(\mathrm{v} / \mathrm{v})$ Triton X-100). Bound proteins were eluted with 750 Elution Buffer (50 mM Tris- $\mathrm{HCl}$ [pH 8.0], $300 \mathrm{mM} \mathrm{NaCl}, 500 \mathrm{mM}$ imidazole). For binding assays 751 containing full length wild type or mutant CLPX, all buffers were supplemented with 2 mM ATP 752 and $10 \mathrm{mM} \beta$-mercaptoethanol. 


\section{3-file00.docx}

753

754

755

756

757

758

759

760

761

762

763

764

765

766

767

768

769

770

771

772

773

774

775

776

777

778

779

780

781

782

783

784

785

786

\section{Cell culturing and treatment}

HeLa cells were cultured in Dulbecco's Modified Eagle's Medium (Life Technologies) supplemented with $10 \%(\mathrm{v} / \mathrm{v})$ fetal calf serum at $37{ }^{\circ} \mathrm{C}$ under an atmosphere of $5 \%(\mathrm{v} / \mathrm{v}) \mathrm{CO}_{2}$. Transfection of plasmid (10 $\mu \mathrm{g}$ ) or 10-20 nM synthetic siRNA (Life Technologies) was performed using Lipofectamine ${ }^{\circledR} 2000$ transfection reagent (Life Technologies) as per the manufacturer's instructions and cells grown for a further $24-72 \mathrm{~h}$, as indicated. For interference of PDIP38 mRNA three independent synthetic siRNA (Life Technologies) were used; Silencer No. 22994 and Silencer Select No. s25055 (s55) and s25056 (s56). The corresponding Silencer Negative Control and Silencer Select Negative Controls No. 1 (nc1) and No. 2 (nc2) were used. For analysis, cells where detached by trypsin treatment $(0.25 \%(\mathrm{w} / \mathrm{v})$ trypsin, $1 \mathrm{mM}$ EDTA; Invitrogen) and washed cell pellets lysed using TC extraction buffer (50 mM Tris-HCl [pH 7.5], $375 \mathrm{mM} \mathrm{NaCl}, 1 \mathrm{mM}$ EDTA, 1\% (v/v) Triton X-100) freshly supplemented with $2 \mathrm{mM}$ phenylmethanesulfonyl fluoride (PMSF). Soluble lysate was collected and used for analysis.

\section{Mitochondrial isolation and manipulation}

Crude mitochondria were isolated from HeLa cells as described ${ }^{12,64}$. In vitro import ${ }^{65}$ was performed at $37{ }^{\circ} \mathrm{C}$ with $\left[{ }^{35} \mathrm{~S}\right] \mathrm{Met} / \mathrm{Cys}-$ labelled preprotein and isolated mitochondria resuspended in Import Buffer (20 mM HEPES-KOH [pH 7.4], $250 \mathrm{mM}$ sucrose, $5 \mathrm{mM} \mathrm{Mg(OAc),} 80 \mathrm{mM} \mathrm{K}(\mathrm{OAc})$, freshly supplemented with $10 \mathrm{mM} \mathrm{Na}$ succinate, $1 \mathrm{mM}$ DTT, $2 \%(\mathrm{w} / \mathrm{v})$ fatty acid free BSA, $5 \mathrm{mM}$ ATP and $5 \mathrm{mM}$ methionine. A mix of valinomycin $(2 \mu \mathrm{M})$ and oligomycin $(10 \mu \mathrm{M})$ was used to dissipate the membrane potential. Following import, mitochondria resuspended in SEM (250 mM sucrose, $1 \mathrm{mM}$ EDTA, $10 \mathrm{mM}$ MOPS-KOH [pH 7.2]) were treated with $\sim 40 \mu \mathrm{g} / \mathrm{ml}$ proteinase $\mathrm{K}$ (Prot. K) for $15 \mathrm{~min}$ at $4{ }^{\circ} \mathrm{C}$. Mitoplasts were formed in 9 parts EM buffer (10 mM MOPS-KOH [pH 7.2], $1 \mathrm{mM}$ EDTA) to 1 part SEM buffer at $4{ }^{\circ} \mathrm{C}$ for $20 \mathrm{~min}$ with gentle pipetting ${ }^{65}$. For protease treatment, mitochondria in SEM buffer, mitoplasts in EM buffer and lysed mitochondria in SEM buffer with $0.5 \%(\mathrm{v} / \mathrm{v})$ Triton X100 were incubated on ice with $50 \mu \mathrm{g} / \mathrm{ml}$ proteinase $\mathrm{K}$ for the times indicated. Proteinase $\mathrm{K}$ was inhibited by the addition of $2 \mathrm{mM}$ phenylmethylsulfonyl fluoride (PMSF) and proteins were immediately precipitated with TCA for analysis.

\section{Immunoprecipitation}

Mitochondrial lysate in immunoprecipitation (IP) Buffer (50 mM Tris-HCl [pH 7.5], $100 \mathrm{mM} \mathrm{KCl,}$ $10 \mathrm{mM} \mathrm{Mg}(\mathrm{OAc}), 5 \% \mathrm{v} / \mathrm{v}$ glycerol) containing $0.5 \%(\mathrm{v} / \mathrm{v})$ Triton X-100, $10 \mathrm{mM}$ ATP and $2 \mathrm{mM}$ phenylmethylsulfonyl fluoride (PMSF) was mixed with Protein A-Sepharose covalently attached to antibodies (anti-PDIP38 or anti-CLPX) by end-over-end rotation for $1 \mathrm{~h}$, at $4^{\circ} \mathrm{C}$. Beads were washed with 3 bed volume (BV) of IP buffer containing $0.25 \%(\mathrm{v} / \mathrm{v})$ Triton X-100, $10 \mathrm{mM}$ ATP and $2 \mathrm{mM}$ PMSF and antibody bound protein eluted using $1 \mathrm{BV}$ of $50 \mathrm{mM}$ glycine [pH 2.5]. 
40922693-file00.docx

\section{Crystallisation, $X$-ray diffraction and structure determination}

788 To investigate the structure of PDIP38, crystal screening was performed and crystals were obtained 789 using 20\% (w/v) PEG8000, $100 \mathrm{mM}$ Hepes, pH 7.5. Crystals of the free and derivatized protein 790 were frozen in liquid nitrogen and data were collected at $100 \mathrm{~K}$ at the Swiss light source (SLS, 791 Villigen, Switzerland; beamline PXII). Data were recorded on a PILATUS 6M detector (Dectris, 792 Baden-Daettwil, Switzerland) and data reduction was performed using the program package XDS

$793 \quad 66,67$. The structure of PDIP38 was solved to $3.1 \AA$ by single anomalous dispersion techniques using 794 one Pt derivative for phasing. The model was refined using PHENIX ${ }^{68}$. Most of the structure was 795 unambiguously assigned in the electron density map except for residues 52-62 at the N-terminus 796 and the loop regions (L3 between residues 108-126) and (L4 between residues 144-167), due to 797 poor density. Supplementary Table 1 provides the statistics for the X-ray data collection and final 798 refined model. All structural figures were generated using ChimeraX_Daily. 
a

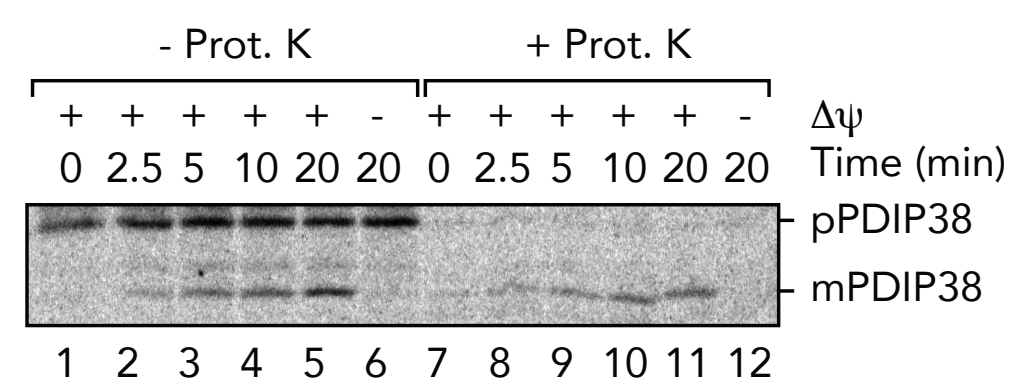

b

$\overbrace{-++++-++++-+++}$ Prot. K $301020303010203030102030 \quad$ Time (min) =-- - - - -

- _ - - TOM20

$-\infty-\infty-\infty \quad-\quad$ TIM23

$--\infty-\infty-\infty-\infty$ CLPP

$\begin{array}{lllllllllllll}1 & 2 & 3 & 4 & 5 & 6 & 7 & 8 & 9 & 10 & 11 & 12\end{array}$
C

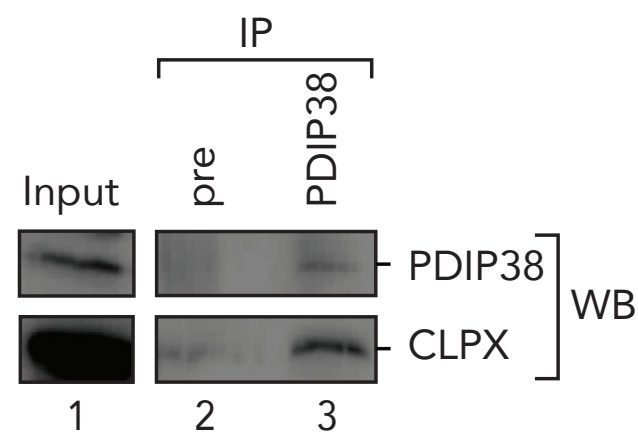

d

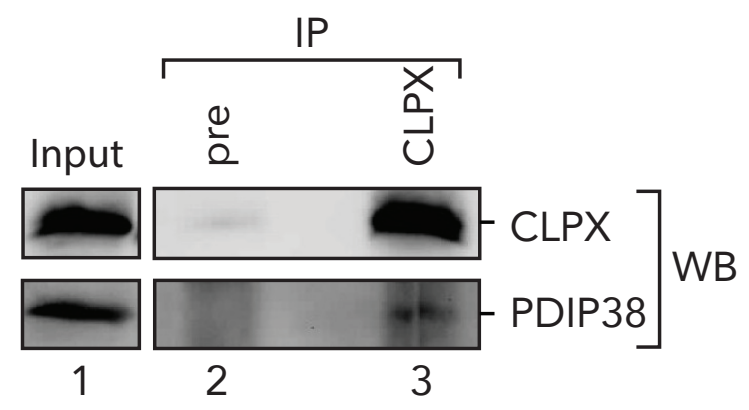


a

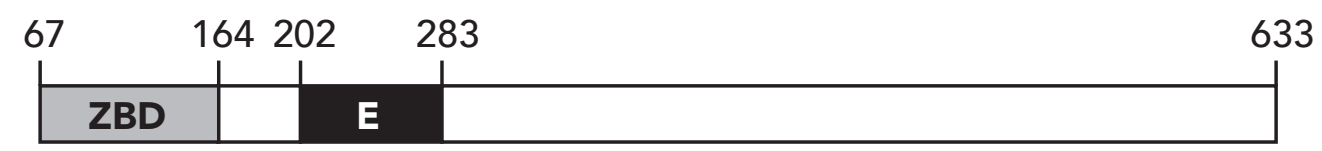

b

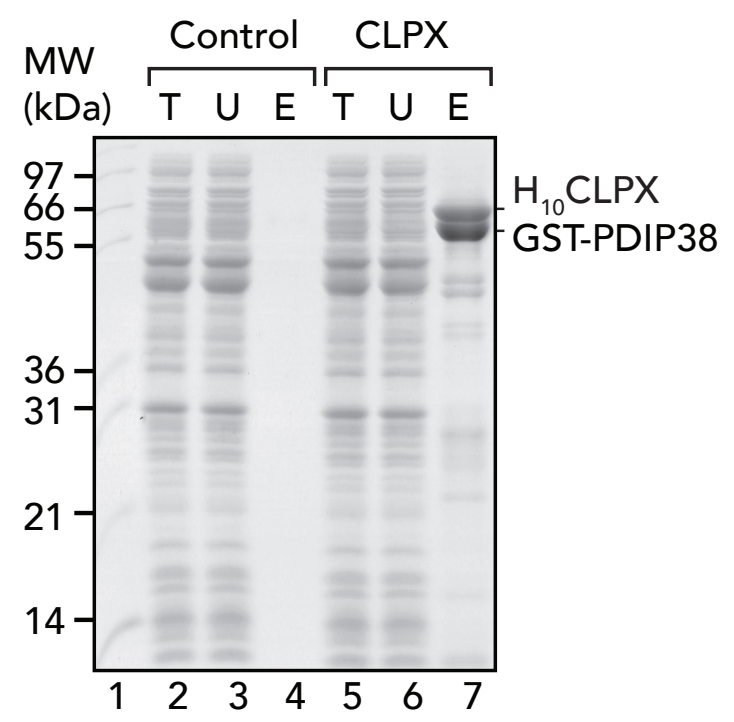

C

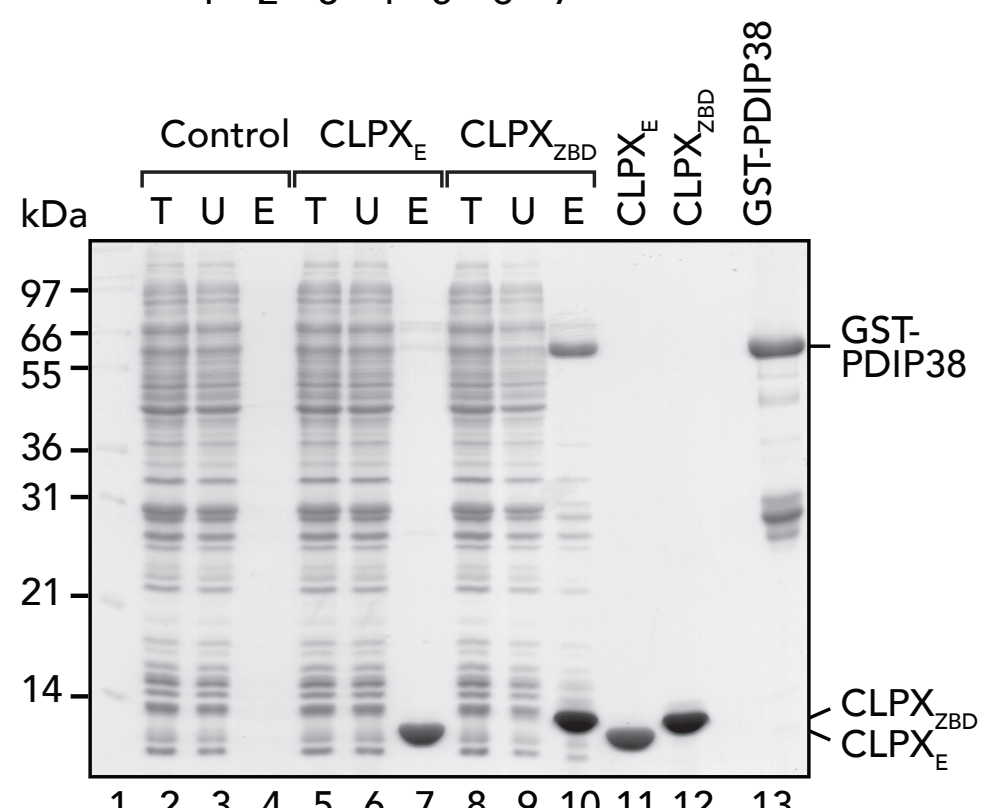

d
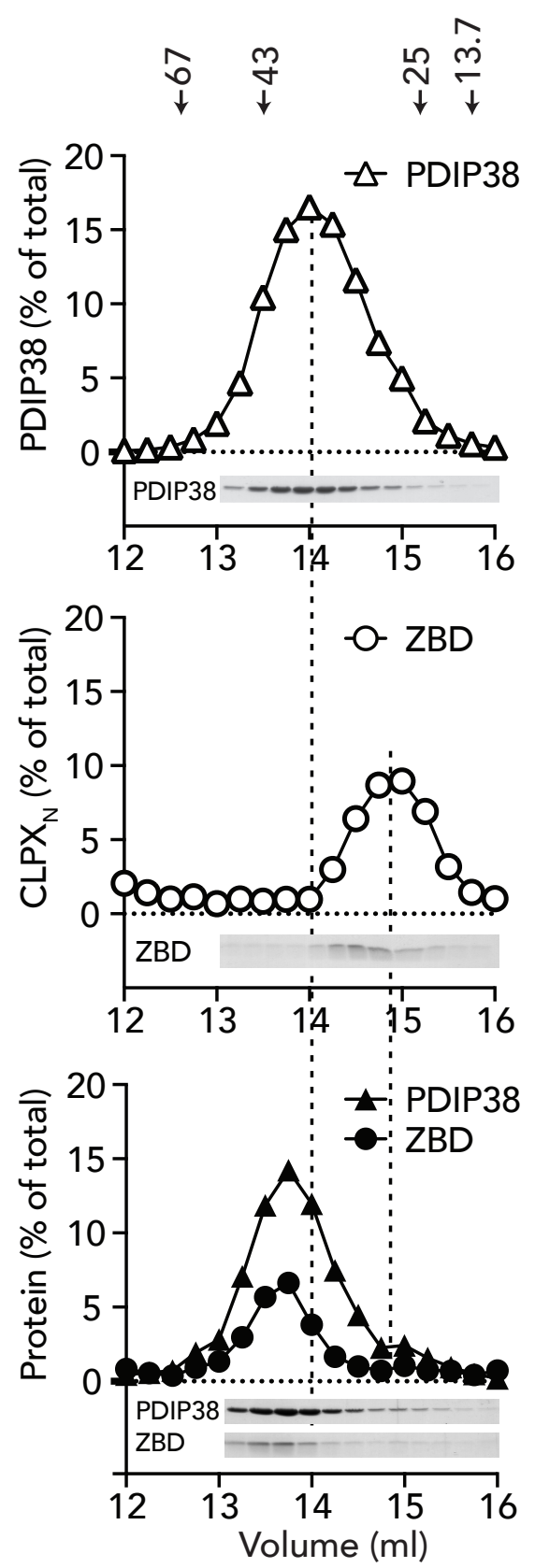

Strack et al., Figure 2 
bioRxiv preprint doi: https://doi.org/10.1101/2020.05.19.105320; this version posted May 22, 2020. The copyright holder for this preprint (which was not certified by peer review) is the authbrnaedemamyights reserved. No reuse allowed without permission.
a $\mathrm{MW}$

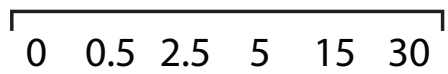
(kDa) $\quad-\quad+\quad+++$ Thermolysin

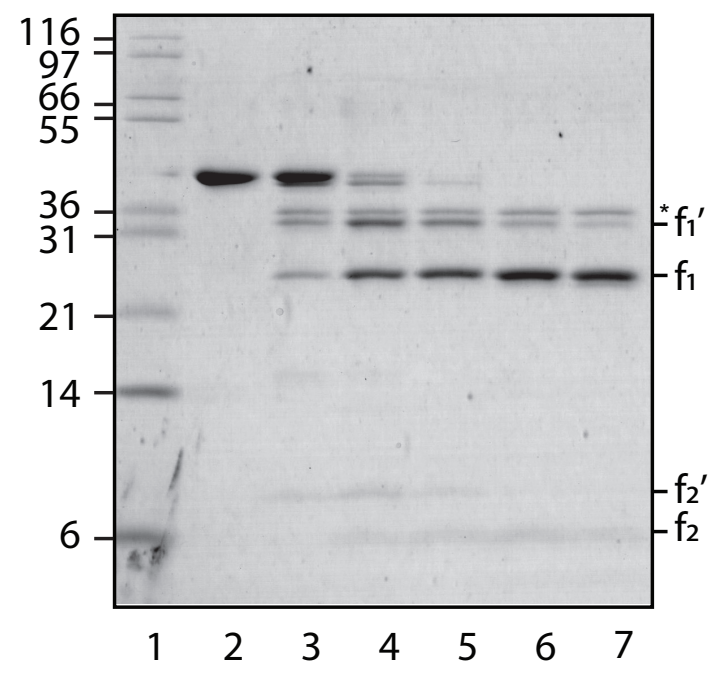

b
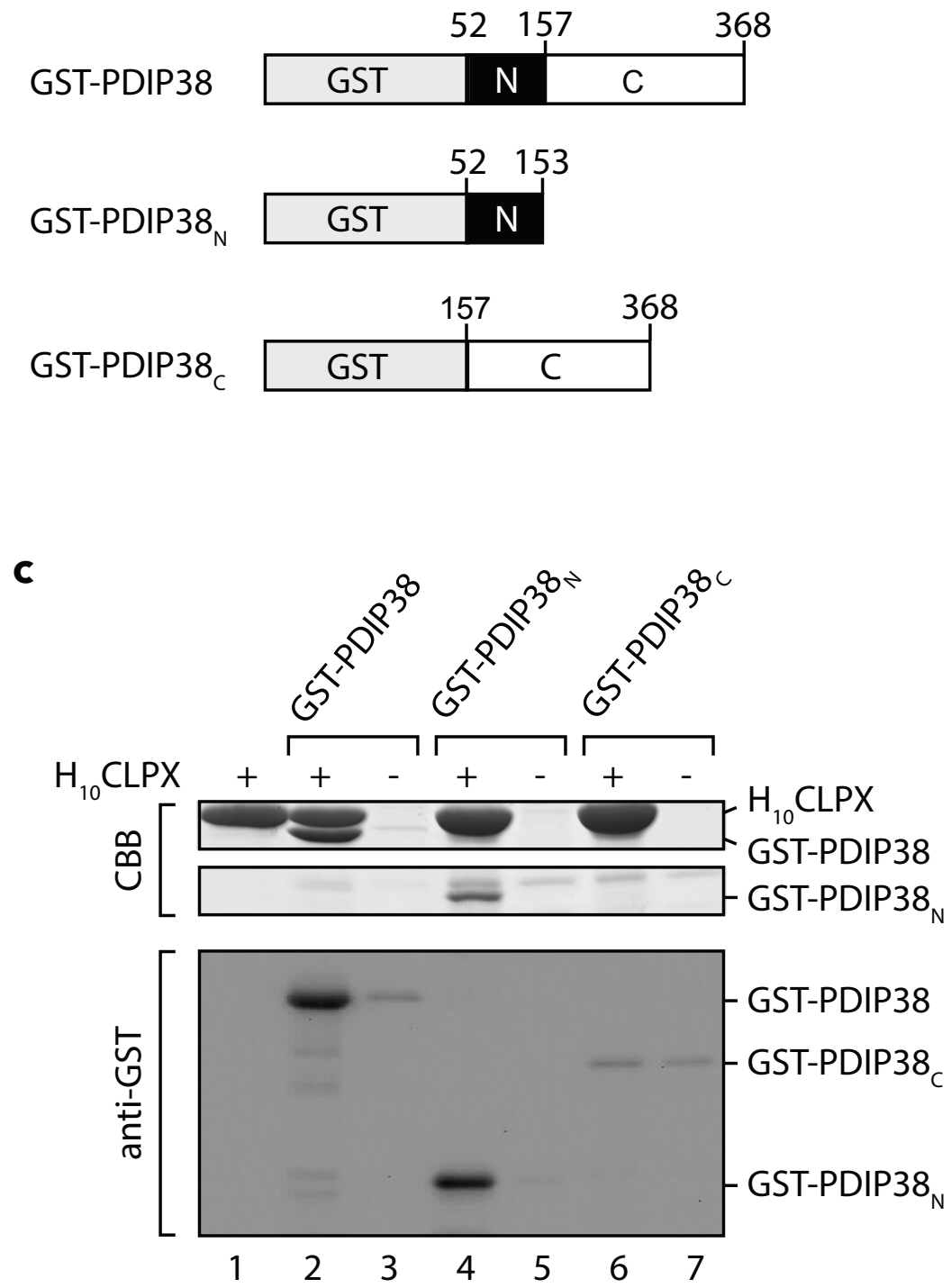


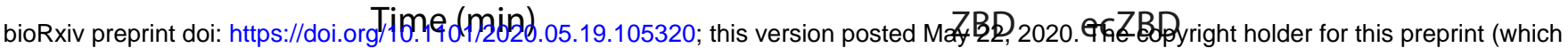
was notcentiffed by peer review) is the authir/funder. All rights reserved. No reyse allowed without permission.

PDIP38 $\begin{array}{llllll}0 & 15 & 30 & 60 & 90 & 120\end{array}$
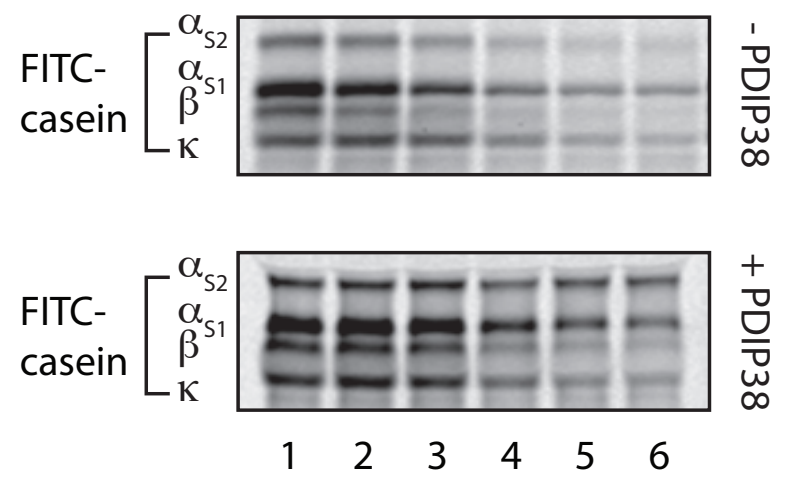

C

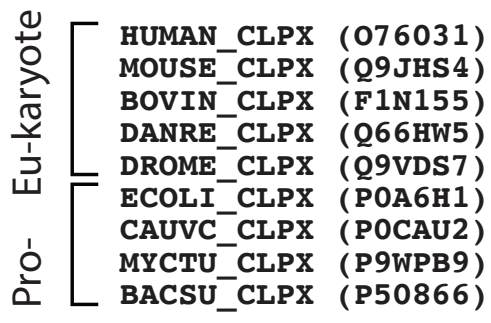

$\mathrm{kDa}+{ }_{-}+{ }_{-}$GST-PDIP38

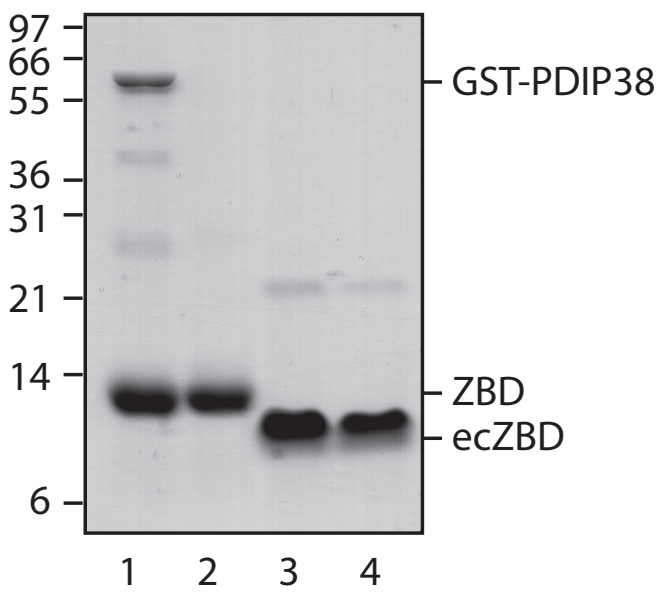

으음:

$\frac{0}{0}$

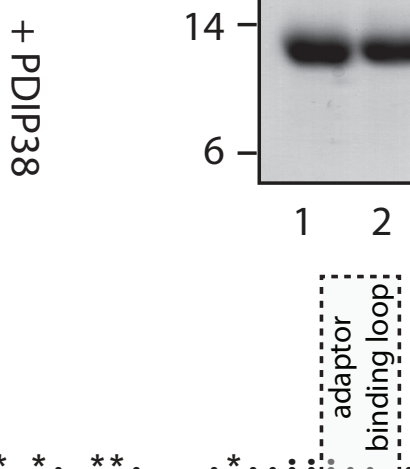

GKGGNQ-LRCPKCGDLCTHVETFV'SSTR'FVKCEKCHHFFV-VLSEA GKGGNO-LRCPKCGDLCTHVETFV'SSTRFVKCEKCHHFFV-VLSEA GKGGNQ-LRCPKCGDLCTHVETFV'SSTRFVKCEKCHHFFV-VLSEA GKGGSQ-LRCPKCGDPCTHVETFV'SSTR'FVKCEKCHHF FV-VLSET GGGSDKFLSCPKCGSACTQVETFV'SSTRFVKCAKCNYFFV-VLSEV GSGKL--LY CSF CGKSQHEVRKLIAG PSIVY I CDECVDLCNDI IREE GDTKST-LYCSFCGKSQHEVRKLI'A GPTVF I CDECVELCMDI IREE GGDL---LKCSFCGKSQKQVKKLI'A GP GVY I CDECIDL CNEI IEEE EKGQ---LKCSFCGKTQDQVRKLV'AGPGVY ICDECIELCTEIVEEE

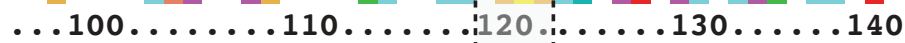

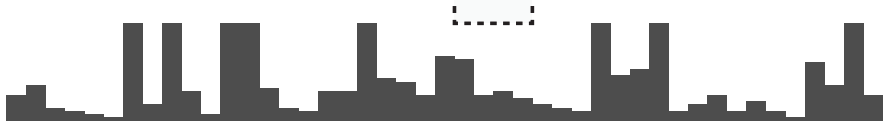

d
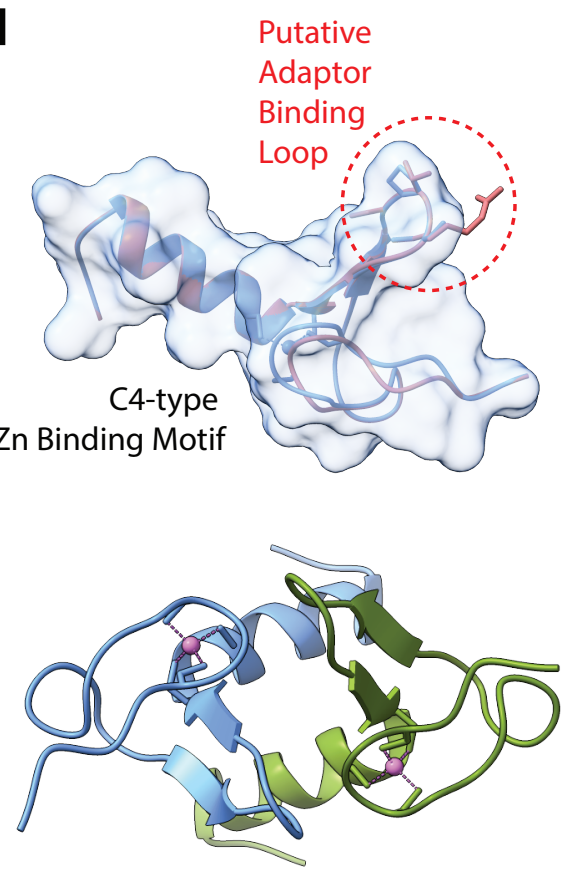

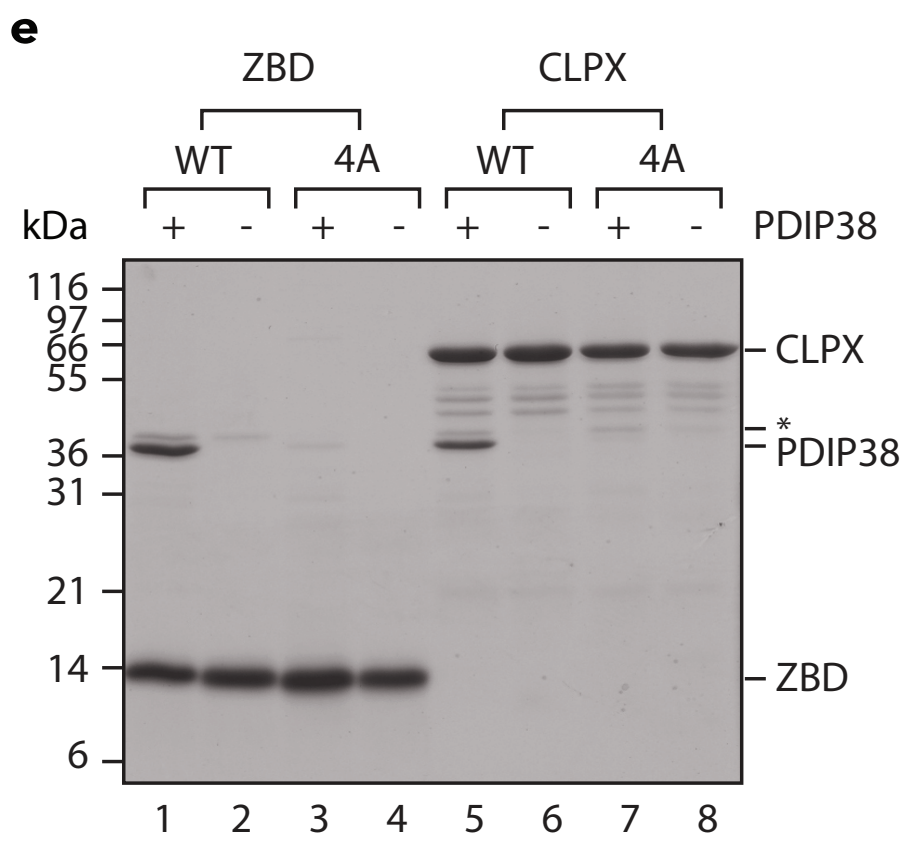


a
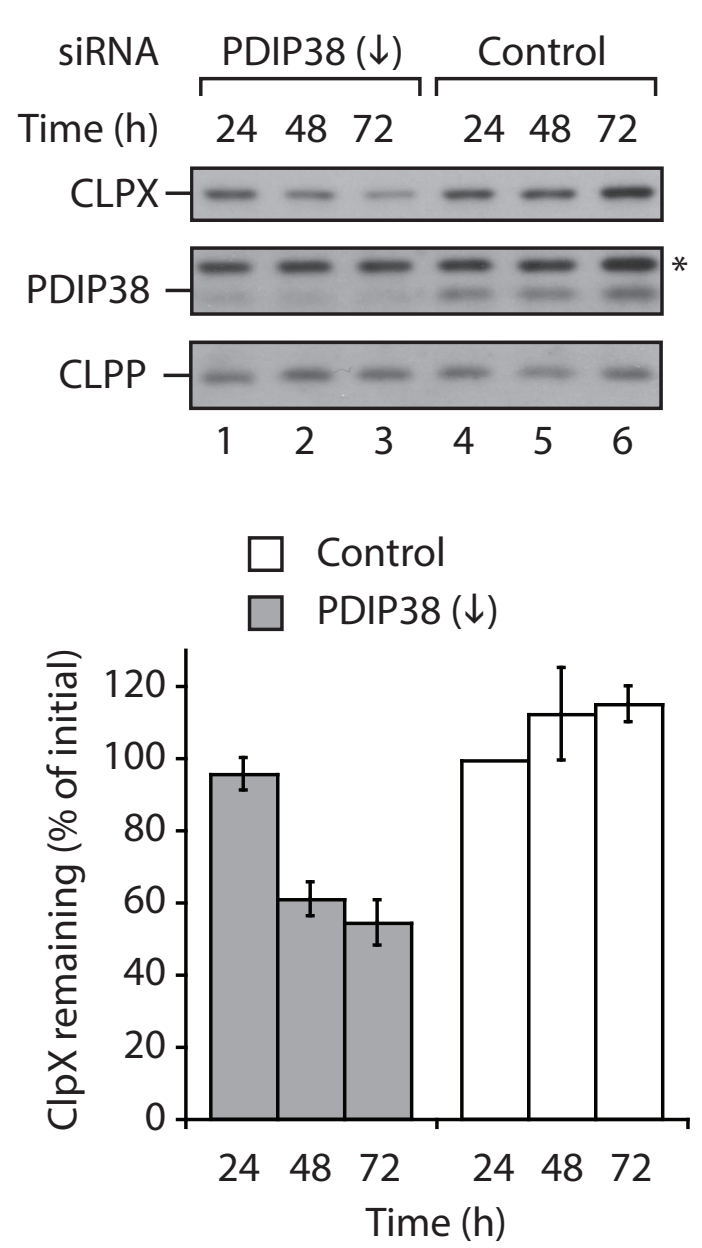

b

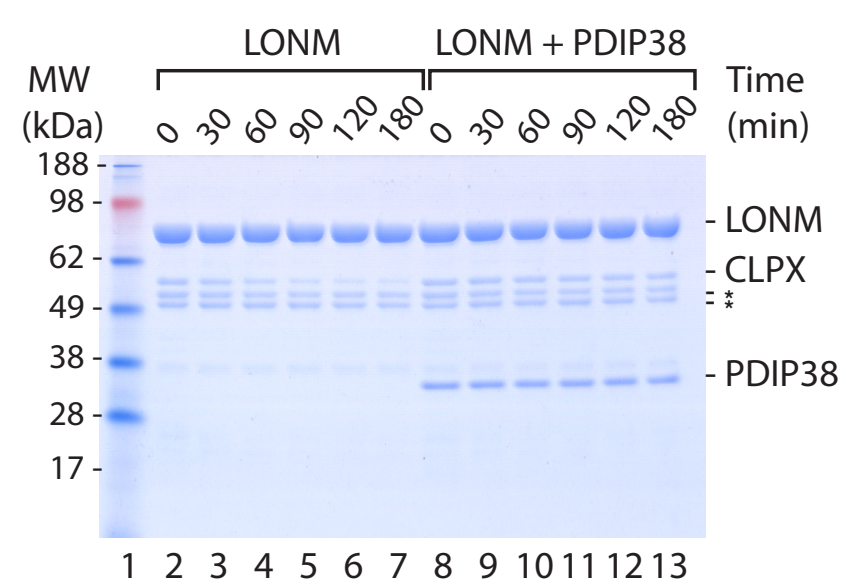

c

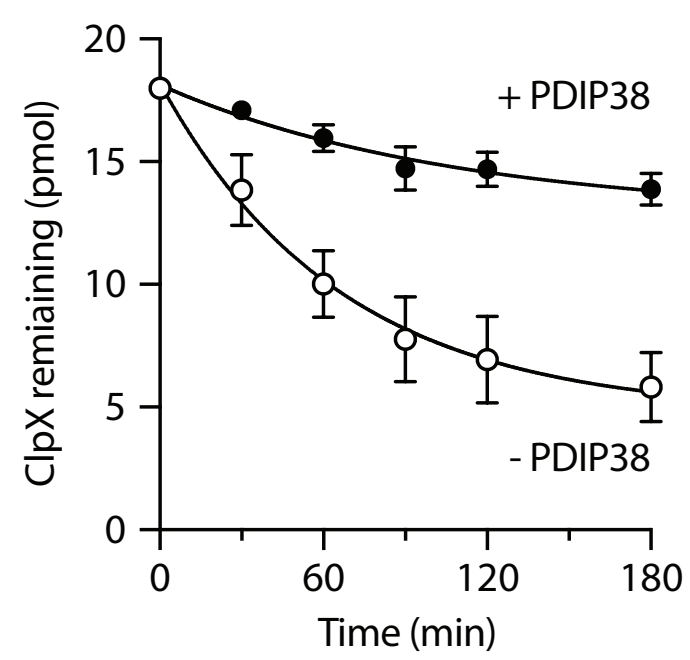

Strack et al., Figure 5 

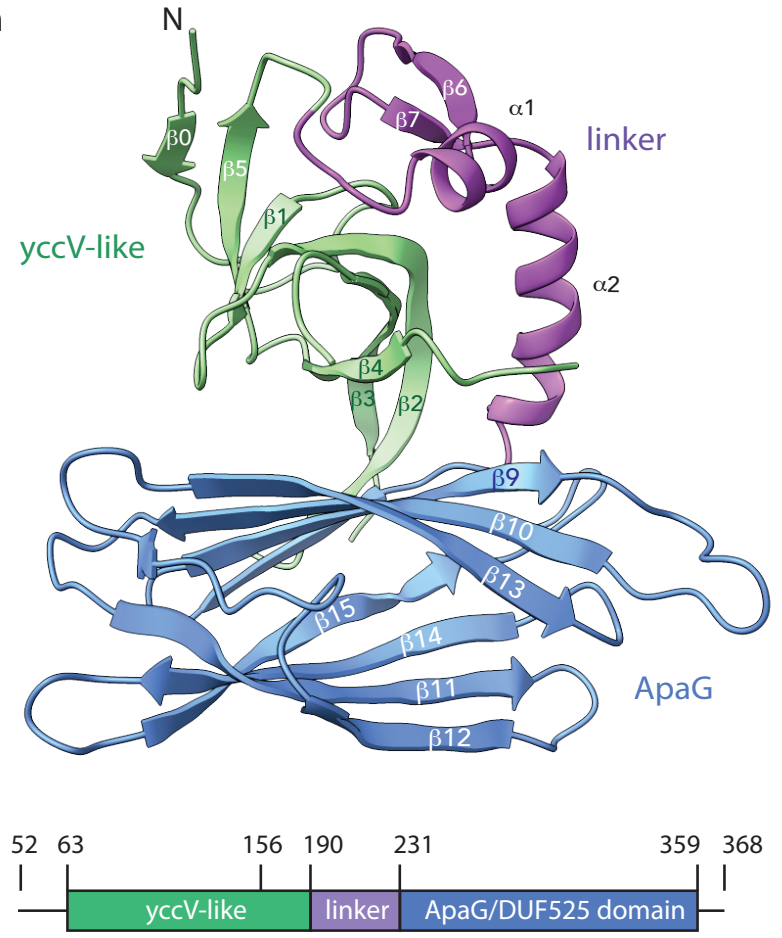

c

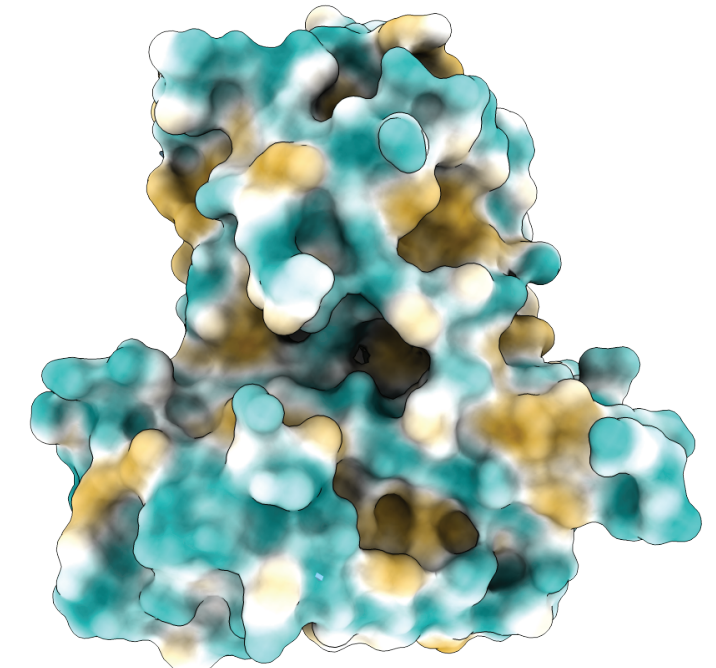

b

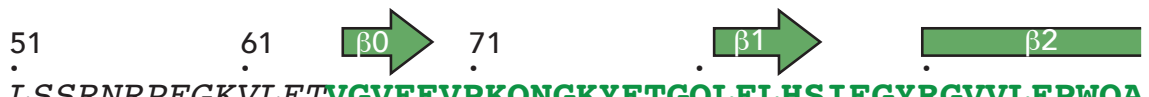
LSSRNRPEGKVLETVGVFEVPKONGKYETGQLFLHS IFGYRGVVLF PWQA

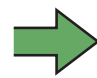
111 121 141

RLYDRDVASA $\dot{A} P E K A E N P A G \dot{H} G S K E V K G K T \dot{H} T Y Y Q V L I D A \dot{R} D C P H I S Q R S$

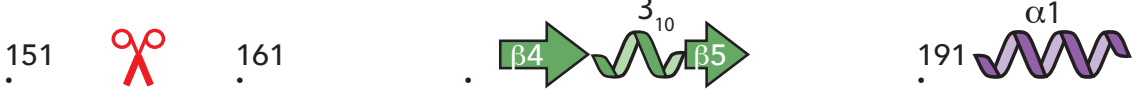
QTEAVTFLANHDDSRALYA I PGLDYVSHEDILPYTSTDQVP IQHELFERF LLYDQTKAPPFVARETLRAWQEKNHPWLELS்VHRETTENIRVTVIPFYM $251 \quad 271 \quad \frac{\beta 11}{\square}>291$ GMREAQNSHVY்WWYCIRLENLDSDVVQLRERHWRIFSLSGTLETVRGRG

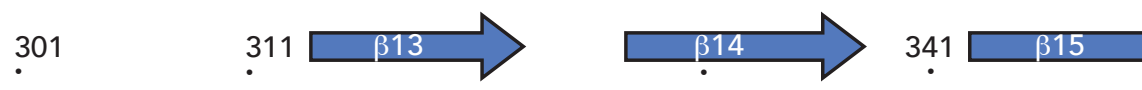
VVGREPVLSKEQPAFQYSSHVSLQASSGHMWGTFRFERPDGSHFDVRIPP $\checkmark \quad 361 \quad 368$

FSLESNKDEKTंPPSGLHن்
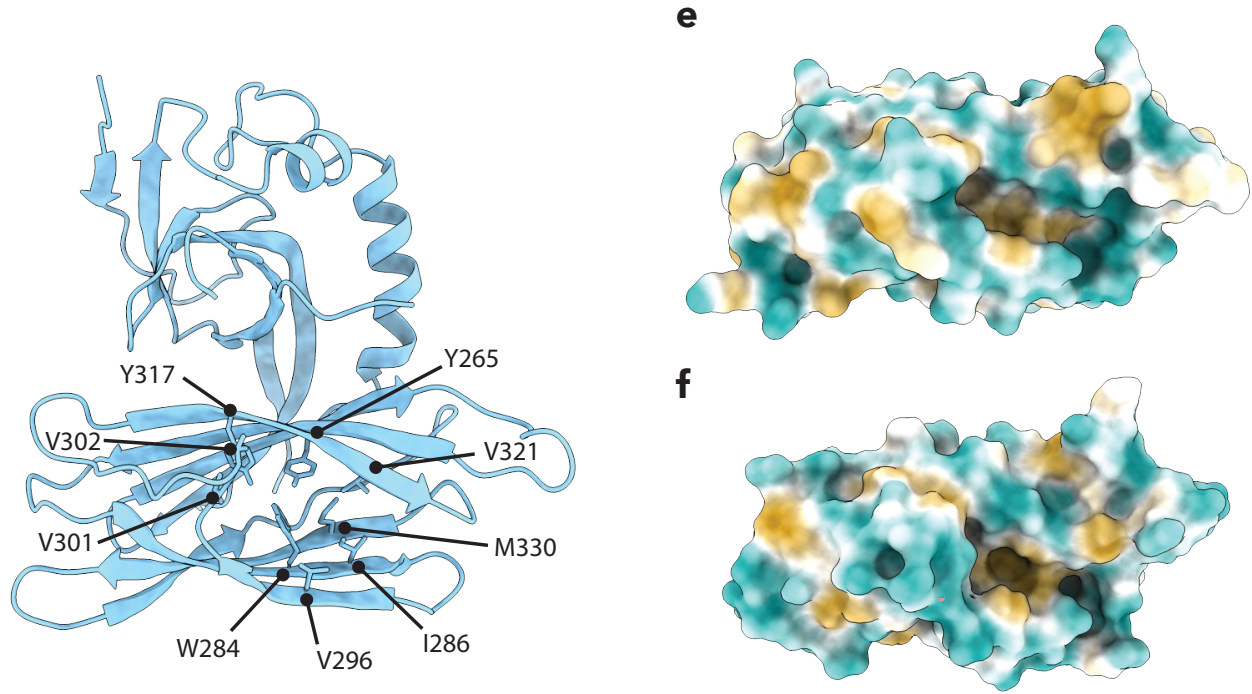

Strack et al., Figure 6 